\title{
D-serine supplement ameliorates MPP+-induced neurotoxicity via attenuating DAPK1-associated pathway in Parkinson's disease models
}

\author{
Wenli Zhang \\ Dalian Medical University
}

\section{Xueying Sun}

Sixth People's Hospital of Dalian

Jun Liu

Dalian Medical University

\section{Yuanwen Peng}

Dalian Medical University

\section{Yuanhua Qin}

Dalian Medical University

\section{Yuefei Xu}

Dalian Medical University

\section{Houli Zhang}

Dalian Medical University

Wenyong Ding ( $\square$ dingwy@dmu.edu.cn )

Dalian Medical University

\section{Sheng Li}

Dalian Medical University

\section{Li Ma}

Dalian Medical University

\section{Research}

Keywords: D-serine, PD (Parkinson's disease), NMDAR (N-methyl-D-aspartate receptor), MPTP/MPP+, DAPK1(death-associated protein kinase 1), calcium, ROS

Posted Date: September 21st, 2021

DOl: https://doi.org/10.21203/rs.3.rs-892540/v1

License: (c) (i) This work is licensed under a Creative Commons Attribution 4.0 International License. Read Full License 
Page 2/31 


\section{Abstract}

Background: D-serine is reported to modulate neurotransmission via regulating the activation of $\mathrm{N}$ methyl-D-aspartate receptor 1 (NMDAR1) in a narrow range, and dysfunction or dysregulation of NMDAR1 contributes to the pathophysiology of Parkinson's disease (PD), a chronic and progressive neurodegenerative disorder. This study aims to further elucidate the action mechanism of Dserine/NMDAR1 in PD.

Methods and Results: At animal level, we found D-Serine and NMDAR1 were cooperatively distributed in mouse brains. Compared to the control mice, a dramatic increase in D-serine content and NMDAR1 expression was revealed in striatum, whereas a significant reduction was found in cortex, hippocampus, cerebellum and brainstem in PD mice. Thus, the tissue-specific D-serine/NMDAR1 was suspected to be associated with PD. Based on the decreased levels of NMDAR1 and D-serine in the MPP ${ }^{+}$-treated glioma cells, a D-serine supplement was introduced. We found that D-serine supplement enhanced NMDAR1 expression, and triggered neuronal cells to be rescued supporting by parkinsonian parameters including morphological observation, a decreased ROS level, an increased dopamine content, and a declined acetylcholine level. Additionally, a decreased calcium, reduced DAPK1 expression, and raised Bcl2 level were found in neuronal cells supplied with D-serine.

Conclusions: We speculated that D-Serine attenuated neuronal cell death via inhibiting DAPK1-related pathway. Additionally, D-serine was confirmed to display an ability to ameliorate the MPTP injury using the MPTP-administrated mice injected with D-serine. Unlike the previous description, D-serine displays a protective effect on neuronal cells. Overall, our finding highlights D-serine as a strong enhancer for NMDAR1 expression and a candidate for PD therapy. This opens up an innovative perspective for neurobiological therapy using $\mathrm{D}$-serine augmentation.

\section{Background}

Nowadays, it is estimated that worldwide population over 65 years of age is approximate $8.5 \%$, and will reach $17 \%$ by 2050 [1]. This astounding number arouses a great concern on neurodegenerative disease such as Parkinson's disease (PD). PD is a chronic and progressive neurodegenerative disorder. Age carries a high risk of Parkinson's disease. PD is characterized by four cardinal motor symptoms including rigidity, bradykinesia, postural instability and tremor, and non-motor symptoms consisting of depression, cognitive decline, anxiety, and sleep behavior disorder, etc.[2-4]. PD was the fastest growing in prevalence, disability, and deaths among neurological disorders. In 2016, 6.1 million (5.0-7.3) individuals was estimated to catch PD globally compared with 2.5 million (2.0-3.0) in 1990, and around 211296 deaths (167771-265160) were caused by PD [5]. Practically, approximately $1 \%$ of persons above the age of 60 years and up to 4 percent of those older than 80 years are affected by this disease [6]. Thus, in order to reduce the disease burden, it becomes more pressing than ever to solve current Parkinson's disease primarily associated with aging. 
Till now, the etiology of Parkinson's disease still remains unknown. Based on the previous description, Parkinson's disease is marked by loss of dopamine-containing neurons in the substantia nigra pars compacta (SNpc) of the midbrain and development of cytosolic Lewy bodies characterized by asynuclein aggregation in the residual dopaminergic neurons $[1,3]$. In addition, gene dysfunction is increasingly recognized to play an important role in the pathogenesis of PD currently. Genotypes derived from a genome-wide association study (GWAS) identify 16 genes which are involved in PD pathogenesis according to single-nucleotide polymorphisms assay [7]. And it is reported that dysfunction or dysregulation of $\mathrm{N}$-methyl-D-aspartate receptor (NMDAR) may contribute to the pathophysiology of PD [8].

NMDAR is an ionotropic glutamate receptor that plays a fundamental role in neuronal communication, synaptic plasticity, synaptic transmission, memory consolidation, and human behavior[8-10]. NMDAR is tetrameric complexes consisting of the two obligatory NR1 subunits (NMDAR1) and two NR2 and/or NR3 subunits $[11,12]$. NR1 subunit, a binding site for glycine/D-serine, regulates NMDAR exit from the endoplasmic reticulum. NR1 serves as ligand-gated cation channels. NR2 and/or NR3 subunits, containing phosphorylation sites critical for NDMAR signaling in response to glutamate, determine the channel permeability to cationic ions including $\mathrm{Na}^{+}, \mathrm{K}^{+}$and $\mathrm{Ca}^{2+}[11,13,14]$.

Opening of the NMDAR ion channel requires the occupation of two distinct binding sites, the glutamate site at NR2/NR3 subunit and the glycine/D-serine site at NR1 subunit [15]. D-serine is supposed to be a physiological co-agonist of NMDAR1 via binding at NR1 subunit. In addition to D-serine, NMDAR1 also binds glycine, but their roles in regulating NMDAR1 activities remain unclear currently. Previous study illustrates that the binding of glycine/D-serine to NMDAR1 appears to be stage-specific. Glycine preferentially activates synaptic NMDAR1 in young mice, but this preference switches to D-serine in adults [16]. D-serine production is catalyzed by serine racemase (SR), which converts L-serine into Dserine in astrocyte [9].

D-serine behaves as a neurotransmitter in the secreted neurons and modulates neurotransmission via regulating NMDAR1 activation. Accordingly, D-serine participates in many pivotal physiological and pathological processes, such as neural development, synaptogenesis, synaptic plasticity, cell migration, neurotoxicity, etc. $[15,17]$. D-Serine is considered as an inductor of neurodegeneration. Since D-serine augments likely NMDAR1-dependent excitotoxicity and over-activation of NMDAR1, it may induce neuronal death. This is supported by numerous studies. For example, during ischemia, the extra cellular D-serine concentration raises in the cerebral cortex, promoting neuronal death mediated by NMDAR1 overactivation; under inflammatory conditions such as trauma, infection, and orischemia, neuronal cells are activated to elevate $\mathrm{D}$-serine levels, promoting an uncontrolled activation of synaptic and extra synaptic NMDAR and inducing neuronal damage; the basal inflammatory conditions of aged individuals may facilitate the abnormal NMDAR activation $[17,18]$. However, there are numerous controversial studies. For example, although D-serine is demonstrated to be the dominant coagonist in NMDAR mediated neurotoxicity in the hippocampus [17], the elevated D-serine levels fully rescues NMDAR-dependent longterm potentiation (LTP) and memory impairments in mice [19]; In addition, changes in D-serine levels in 
the brains of patients with Alzheimer's disease (AD) have been controversial. Previous study documents a lower level of D-serine in the cortices of AD patients, whereas another study illustrates an elevated Dserine level in the cerebrospinal fluid of AD patients compared with healthy controls [20]. NMDAR antagonists lead to NMDAR hypofunctionality throughout the brain, and the animal models with schizophrenia not to target NMDARs in a neural circuit specific manner, thus to fail in precisely recapitulating pathophysiology of schizophrenia. Therefore, Olney et al. argue whether some NMDAR antagonists can generate neurotoxicity, although neurotoxicity is evidenced by neuronal necrosis, neuronal vacuolization, and other cytotoxic changes $[12,21]$. Additionally, recent study illustrates that the concentration of D-serine maintains in a narrow range in vivo, and its desregulation may lead to pathology. Aberrantly elevated D-serine levels are associated with NMDAR-mediated neurotoxicity, whereas abnormally reduced D-serine levels are related to hypofunctions of synaptic plasticity and deficits of memory [17].

Actually, the association of D-serine with NMDAR1 and physiological roles of D-serine are still under debate currently. And the mechanism of D-serine participated in PD is scantly known. In this study we aimed to investigate whether D-serine and NMDAR1 levels were altered in the various specialized structures of mouse brains with PD, and elucidate the association D-serine with NMDAR1 and relationship of D-serine/NMDAR1 with PD pathogenesis, further to unravel the mechanism of Dserine/NMDAR1 involved using D-serine supplied to cultured astrocytes and MPTP-administrated mice.

\section{Methods}

\section{Ethical approval}

This study was carried out in accordance with the principles of the Basel Declaration and recommendations of Dalian Medical University for laboratory animals. The protocol was approved by the Animal Ethics Committee of Dalian Medical University.

\section{Cell culture, treatment and cell viability assay}

Cells and cell culture: Rat C6 glioma cells, purchased from the Cell Bank of Type Culture Collection of Chinese Academy of Sciences (Shanghai, China), were cultured in Dulbecco's modified Eagle's medium (DMEM) supplemented with $1 \%$ glucose, $10 \%$ fetal bovine serum (FBS), and 100 units $/ \mathrm{ml}$ of penicillin/streptomycin (50 $\mu \mathrm{g} / \mathrm{ml}$ penicillin: $50 \mu \mathrm{g} / \mathrm{ml}$ streptomycin=1:1). Cells were routinely grown at $37^{\circ} \mathrm{C}$ in a humidified incubator containing $5 \%$ carbon dioxide.

Drug treatment: To induce cells with PD-like neurotoxicity, rat C6 glioma cells were exposed to medium containing $\mathrm{MPP}^{+}(1 \mathrm{mmol} / \mathrm{L}, 1.5 \mathrm{mmol} / \mathrm{L}, 2 \mathrm{mmol} / \mathrm{L}, 2.5 \mathrm{mmol} / \mathrm{L}, 5 \mathrm{mmol} / \mathrm{L}$ and $7.5 \mathrm{mmol} / \mathrm{L}$ respectively; Sigma, USA) for another $24 \mathrm{~h}$ after they were normally grown for $24 \mathrm{~h}$. The control cells were grown under the same conditions in the absence of MPP ${ }^{+}$. 
MTT assay: The viability of rat $\mathrm{C} 6$ glioma cells treated with $\mathrm{MPP}^{+}$was assessed using a colorimetric MTT (3-[4,5-dimethylthiazol-2-yl] -2,5-diphenyltetrazolium bromide) assay. After the rat $\mathrm{C} 6$ glioma cells were treated with MPP+, $100 \mu \mathrm{l}$ of MTT solution $(0.5 \mathrm{mg} / \mathrm{mL}$ in phosphate buffered saline) were added, and the cells were cultured at $37^{\circ} \mathrm{C}$ with $5 \% \mathrm{CO}_{2}$ for $4 \mathrm{~h}$. Then, $50 \mu \mathrm{LMSO}$ was added into cells to dissolve the purple-blue MTT formazan crystals at $37^{\circ} \mathrm{C}$ with $5 \% \mathrm{CO}_{2}$ for $40 \mathrm{~min}$. The absorbance at 490nm was measured using a spectrophotometer (Thermo Fisher Scientific, USA). The cell viability, indicated by the net absorbance, was expressed as the percentage compared to the controls which were assumed as $100 \%$.

Morphological analysis: The morphology of rat C6 glioma cells was observed using a light microscope (Olympus, Japan).

\section{Preparation of protein lysates}

PD mouse model was established as described previously [22]. On the 7th day after the last MPTP (1Methyl-4-phenyl-1,2,3,6-tetrahydropyridine; Sigma, USA) injection, the mice were sacrificed by cervical dislocation. The various specialized structures of mouse brain, including cortex, cerebellum, brain stem, striatums, midbrain, and hippocampus, were dissected carefully. After washing with ice-cold $0.9 \%$ physiological saline, they were homogenized in ice cold RIPA lysis buffer [50 mmol/L Tris (pH7.4) containing $150 \mathrm{mmol} / \mathrm{L} \mathrm{NaCl}, 1 \%$ Triton X-100, 1 \% sodium deoxycholate, $0.1 \%$ SDS and $1 \mathrm{mmol} / \mathrm{L}$ PMSF] and cleared at $14000 \mathrm{rpm}$ for $30 \mathrm{~min}$ twice. For cells, the cultured rat C6 glioma cells in a $10 \mathrm{~cm}$ dish were digested with trypsin, and collected by centrifugation at $1000 \mathrm{rpm}$ for $5 \mathrm{~min}$. Then, the rat $\mathrm{C} 6$ glioma cells were broken by ultrasonication ( $5 \mathrm{~S}$ on, $3 \mathrm{~S}$ off) followed by clearance via centrifugation at $14000 \mathrm{rpm}$ for $30 \mathrm{~min}$ twice. Subsequently, the protein lysates were quantified using a BCA kit (keyGEN $\mathrm{BioTECH}$, China) followed by storage at $-80^{\circ} \mathrm{C}$ for a further investigation.

\section{Detection of DA and D-Serine contents analyzed by High Performance Liquid Chromatography}

The level of D-Serine and dopamine (DA) were measured by performing reverse phase high performance liquid chromatography (RP-HPLC). After quantification and normalization of protein concentrations, the prepared protein lysates from the various specialized structures of mouse brains and rat C6 glioma cells were allowed to remove high-abundance proteins by adding equal volume of cold acetone.

Derivatization procedure: For D-Serine detection, the deproteinated lysates were required to be derivatized prior to HPLC separation. The derivatization working solution were prepared daily by dissolving $10 \mathrm{mg}$ ophthaldialdehyde (OPA; Sigma, USA) and $10 \mathrm{mg} \mathrm{N}$-acetyl-L-cysteine (NAC; Sigma, USA) in $1 \mathrm{ml}$ absolute ethanol, and adding $0.2 \mathrm{~mol} / \mathrm{ml}$ sodium tetraborate decahydrate (borate, $\mathrm{Na}_{2} \mathrm{~B}_{4} \mathrm{O}_{7}$, adjusted to $\mathrm{pH} 9.8$ with $5 \mathrm{~mol} / \mathrm{L} \mathrm{NaOH}$ ) to the final volumes of $5 \mathrm{ml}$. The OPA/NAC working solution was stored in covered plastic vials at $4^{\circ} \mathrm{C}$ in 4 hours. Stock D-Serine standard was prepared by dissolving D-Serine in deionized water at a concentration of $1 \mathrm{mg} / \mathrm{ml}$ and stored at $4^{\circ} \mathrm{C}$ for up to 1 month. Standard dilutions were made fresh daily in borate buffer. The derivatization procedure consisted of D-Serine standard/the deproteinated samples 
and OPA/NAC working solution $(1: 1, \mathrm{v} / \mathrm{v})$. The reaction mixtures were manually mixed and incubated at room temperature in darkness. Then, the derivatized solution was filtered using a $0.22 \mu \mathrm{m}$ nylon filter with a syringe and injected for HPLC analysis $(20 \mu \mathrm{l})$.

HPLC conditions: Chromatographic separation was performed at $25^{\circ} \mathrm{C}$ on a C18 column $(3.9 \mathrm{~mm} \times 150$ $\mathrm{mm}$, Thermo Fisher Scientific, USA). For D-serine analysis, the mobile phase was a mixture of ammonium acetate buffer ( $50 \mathrm{mmol} / \mathrm{L}, \mathrm{pH} 6.0$, eluent $\mathrm{A})$ and methanol (95:5, v/v; eluent $\mathrm{B})$; the flow rate was 1 $\mathrm{ml} / \mathrm{min}$ over the course of HPLC separation; the fluorescence detector was set up at 350nm for excitation and at $450 \mathrm{~nm}$ for emission, and calibration curves of D-Serine were established in the range of $1 \mathrm{ng} / \mathrm{ml}-$ $10 \mu \mathrm{g} / \mathrm{ml}$. For DA analysis, the mobile phase consisted of acetic acid buffer ( $\mathrm{pH} 3.5$, containing 12 $\mathrm{mmol} / \mathrm{L}$ acetic acid, $0.26 \mathrm{mmol} / \mathrm{L}$ EDTA disodium, eluent A) and methanol (86:14, v/v; eluent B); the flow rate was $0.5 \mathrm{ml} / \mathrm{min}$; DA detection was monitored by the absorbance at the wavelength of $280 \mathrm{~nm}$; calibration curves of DA were established in the range of $1 \mathrm{nmol} / \mathrm{L}-10 \mu \mathrm{mol} / \mathrm{L}$. The data were collected and analyzed via the automation system software. All experiments were run at minimum in triplicate.

\section{Western blot analysis (WB)}

Protein lysates from the various specialized structures of mouse brains or rat $\mathrm{C} 6$ glioma cells was run on a SDS-acrylamide gel electrophoresis (SDS-PAGE) in a vertical electrophoresis apparatus and transferred to a PVDF membrane in blotting buffer $(20 \mathrm{mmol} / \mathrm{L}$ Tris-base, $150 \mathrm{mmol} / \mathrm{L}$ glycine and $20 \%$ methanol) to make proteins accessible to the detection by monoclonal antibodies [23]. The commercially available monoclonal antibodies including anti-GAPDH antibody (Abcam, England), anti-Bcl2 antibody (Abcam, England), anti-DAPK1 antibody (Abcam, England), anti-TH antibody (Santa Cruz Biotechnology, USA) and anti-NMDAR1 antibody (Sigma, USA) were employed in this study. The signals of protein bands were detected using an enhanced chemiluminescent (ECL) system (GE Healthcare Bio-Sciences Corp.).

\section{Immunohistochemistry (IHC)}

Whole mouse brains were saturated in $15 \%$ picric acid in PBS followed by storage in $20 \%$ sucrose in PBS at $4{ }^{\circ} \mathrm{C}$ for IHC assessment as described previously [22]. After being blocked with $2 \%$ bovine serum albumin (BSA, Sigma, MO) for $1 \mathrm{~h}$ at room temperature, tissue slices were incubated with primary antibodies of anti-NMDAR1 for $2 \mathrm{~h}$, and Peroxidase-linked secondary antibodies in order.

Diaminobenzidine (DAB) was used as a chromogen for visualization of immunolabeling. The sections were then counterstained with hematoxylin. After counterstaining, the slices were dehydrated using a gradient concentration of alcohol including $75 \%, 85 \%, 95 \%, 100 \%$ for 6 min respectively, and were then immersed in xylol for $5 \mathrm{~min}$. After being dried in an incubator at $55^{\circ} \mathrm{C}$, images of stained specimens were captured using a light microscope. The endogenous peroxidase activity was neutralized using the peroxidase block in this study.

\section{Immunocytochemistry (ICC)}


Rat C6 glioma cells grown on cover slips in 24-well plate were fixed with pre-warmed $4 \%$ paraformaldehyde in phosphate-buffered saline (PBS) for $10 \mathrm{~min}$. After being washed three times in PBS, cells were then permeabilized with 1\% Triton X-100 in PBS for 10 min. The immunocytochemical staining was then routinely conducted. The slides were incubated with $2 \%$ BSA for $1 \mathrm{~h}$. Each section was then incubated for $2 \mathrm{~h}$ with primary antibodies of anti-NMDAR1 diluted in blocking solution ( $2 \% \mathrm{BSA}$ in PBS). The slides were then incubated with the HRP-conjugated second antibody for 40 min, and DAB was employed as a chromogen. The slides were counterstained with hematoxylin. The endogenous peroxidase activity was neutralized using the peroxidase block, and an inverted light microscope was used for capturing images. The operation was conducted at room temperature.

\section{Determination of cellular Acetylcholine content}

Acetylcholine (ACh) content in rat $\mathrm{C} 6$ glioma cells was determined using commercially available ACh quantitation kit, and performed according to the manufacturer's instructions (Sigma, USA). The absorbance of ACh was measured using a microplate reader at $550 \mathrm{~nm}$.

\section{Cell apoptosis analyzed by Flow cytometric}

Cell apoptosis was analyzed using flow cytometry and Annexin V-FITC Apoptosis Detection Kit (Jiangsu Kaiji Biotech, China). The rat $\mathrm{C} 6$ glioma cells were seeded in 24-well culture plates for $24 \mathrm{~h}$ and then treated with $\mathrm{MPP}^{+}$for another $24 \mathrm{~h}$. After treatment with $\mathrm{MPP}^{+}$, the $\mathrm{C} 6$ glioma cells were treated with various intervention reagents for another $24 \mathrm{~h}$. After being washed twice with PBS, the treated cells were stained with dual staining Annexin V-fluorescein isothiocyante (FITC)- propidium iodide (PI) at room temperature in the dark for $15 \mathrm{~min}$. Subsequently, the stained cells were measured by flow cytometry (Beckman-CytoFLEX Coulter, USA). The data were analyzed by FlowJo (BD Biosciences, USA), and the apoptotic cells were counted and presented as a percentage of the total cell counts. The analysis was performed in triplicate.

\section{Measurement of total intracellular calcium}

Total intracellular calcium ( $\left.\left[\mathrm{Ca}^{2+}\right]_{\mathrm{cyt}}\right)$ was measured using the fluorescent probe Fluo-3AM (1-[2-Amino-5(2,7-dichloro-6-hydroxy-3-oxo-9-xanthenyl)phenoxy]-2-(2amino-5-methylphenoxy)ethane-N,N,N, $\mathrm{N}^{\prime}$ tetraaceticacid,pentaacetoxymethyl ester) which is a calcium indicator. Fluo-3AM exhibits an increase in fluorescence signals upon binding $\mathrm{Ca}^{2+}$. The rat $\mathrm{C} 6$ glioma cells were seeded in 96-well culture plates for $24 \mathrm{~h}$, then exposed to $\mathrm{MPP}^{+}$for $24 \mathrm{~h}$ and their corresponding reagents for another $24 \mathrm{~h}$ sequentially. The treated rat C6 glioma cells were loaded with Fluo-3AM $(1: 5000, v / v)$ for $30 \mathrm{~min}$ at $37^{\circ} \mathrm{C}$. The cells were subsequently rinsed twice with PBS for removing the excess dye. The fluorescence spectrophotometer was used for the fluorometric measurement of intracellular $\left[\mathrm{Ca}^{2+}\right]$. The excitation wavelengths for Fluo$3 \mathrm{AM}$ were $488 \mathrm{~nm}$ with emission at $525 \mathrm{~nm}$. The experiments were at minimum carried out three times independently, and the $\left[\mathrm{Ca}^{2+}\right]_{\mathrm{cyt}}$ values were derived from luminometric units.

\section{Detection and quantification of intracellular ROS}


The intracellular reactive oxygen species (ROS) was measured using 2',7'-dichlorofluorescin diacetate (DCFH-DA; Sigma-Aldrich) as a fluorescent probe. DCFH-DA can be oxidized to the highly fluorescent dichlorofluorscein (DCF) which can freely permeate cell membranes and is extensively used to monitor ROS production. The rat $\mathrm{C} 6$ glioma cells were seeded in black $96-$ well plates with a clear bottom. After exposure to $\mathrm{MPP}^{+}$and their corresponding reagents for another $24 \mathrm{~h}$ sequentially, the treated cells were incubated with DCFH-DA $(10 \mu \mathrm{mol} / \mathrm{L})$ at $37^{\circ} \mathrm{C}$ for $30 \mathrm{~min}$. After the cells were rinsed with PBS twice, the fluorescence intensity was assessed by a fluorescence microplate reader with an excitation wavelength of $485 \mathrm{~nm}$ and an emission wavelength of $535 \mathrm{~nm}$.

\section{D-serine administration and neurobehavioral testing}

The MPTP-administrated mice were randomly divided into three groups: MPTP group $(n=4)$, MPTP/Dserine group $(n=4)$ and MPTP/Levodopa group $(n=4)$. Mice were performed by administering intraperitoneal injections of D-serine (0.3 mg/ $\mathrm{kg}$ D-serine dissolved in physiological saline), at once for each day in the MPTP/D-serine group, mice were intraperitoneally injected with Levodopa/Benzylhydrazine (4:1, $12 \mathrm{mg} / \mathrm{kg}$ Levodopa dissolved in physiological saline), at twice for each day in the MPTP/Levodopa group, while the mice in the MPTP group received the same volume of physiological saline $(10 \mathrm{ml} / \mathrm{kg})$ via intraperitoneal injection.

Neurobehavior of mice were observed and recorded each week by means of horizontal grid test and vertical grid test. Horizontal grid test was measured using a horizontally standard wire where the mouse was placed, facing upward. Duration of hanging on the wire was monitored for each mouse in three independent trials with an interval of $20 \mathrm{~min}$ [24]. Vertical grid test was based on a vertical wooden pole (50 $\mathrm{cm}$ in length and $1.5 \mathrm{~cm}$ in diameter) with a rough surface [25]. Mouse was placed head down on the top of role, and the latency for mouse to climb down the pole from the top to the base was measured as the average of three trails. Trials were considered a failure if the mouse slid down or jumped the pole.

\section{Image quantification and statistical analyses}

The protein bands on Western blot images were quantified using Image $\mathrm{J}$ (version 1.42). The color images were converted to gray-level intensity for quantification, and slight variations in background staining were corrected by subtracting background density. Quantification of the immunohistochemistry images was conducted using Image-Pro Plus software (version 6.0).

Statistical comparisons were undertaken using GraphPad Prism 6 and SPSS software package (version 21). A two-tailed paired $t$ test was used for comparisons between two independent groups. Data were presented as means \pm SEM, and statistical significance was set at the level of $5 \%(P<0.05)$.

\section{Results}

\section{Establishment of the successful PD mouse model}


In this study, C57BL/6 male mice were utilized for establishing the mouse model with PD. MPTP was intraperitoneally administered to the mice as an inducer of PD. Due to the obtained evidences including the obvious motor impairments, a significant reduction in striatal DA and 5-HT content, and mesencephalic TH abundance, the PD mouse model was substantially confirmed to be established by intraperitoneal injection with MPTP as described recently [22, 26].

\section{A tissue-specific expression of NMDAR1 was detected in the mice of MPTP-administrated group}

To evaluate the changes of NMDAR1 abundance in the various specialized structures of the MPTPadministrated mouse brains, including cortex, cerebellum, brain stem, striatums, midbrain, and hippocampus, Western Blot analysis separated by a $10 \%$ SDS-PAGE and IHC were performed using monoclonal anti-NMDAR1 antibody as the probe.

Western blot analysis: Western blot analysis identified NMDAR1 protein at the molecular weight (MW) of 100冈130 kDa as shown in Fig. 1a. The size of the detected bands was consistent with the predicted NMDAR1 of $120 \mathrm{kDa}$. Fig. 1a also reveals a changeable abundance of NMDAR1 in the brains of the MPTP-administrated mice. As determined by Western blot analysis, the optical density ratio of NMDAR1 to GAPDH was statistically analyzed using Image J. As shown in Fig. 1b, comparison analyses revealed a significant decrease in NMDAR1 expression in the structures of cortex (Control: 1.073 \pm 0.136 ; PD: 0.598 $\pm 0.104 ; P=0.009$ ), cerebellum (Control: 1.945 $\pm 0.127 ; P D: 1.129 \pm 0.109 ; P=0.001)$, brain stem

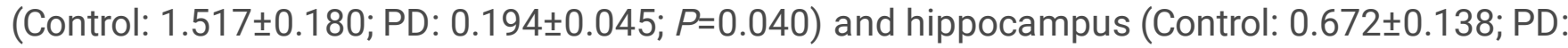
$0.325 \pm 0.104 ; P=0.026)$ in the MPTP-administrated groups compared to their controls. Additionally, a

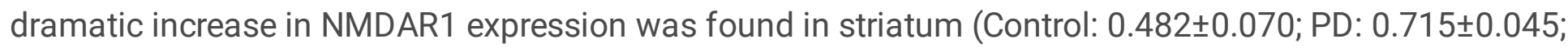
$P=0.008$ ), and a slight increase in NMDAR1 expression was detected in midbrain (Control: 0.981 \pm 0.068 ; PD: $1.054 \pm 0.084 ; P=0.305)$ in the MPTP-administrated PD group relative to the control. However, no significant difference of NMDAR1 abundance was found in midbrain between the MPTP-administrated group and its control.

IHC examination: To further confirm the tissue-specific expression of NMDAR1 in the structures of mouse brains obtained from Western blot analysis, IHC was conducted in this study as shown in Fig. 1d. The signals with dark-stained dot-like structures were scored as positive in this study, whereas as negative. Fig. 1d demonstrates that mouse brains display a strong immunoreactivity to the monoclonal antiNMDAR1 antibody. Statistic analysis on the percentage of positive areas in the IHC images revealed a

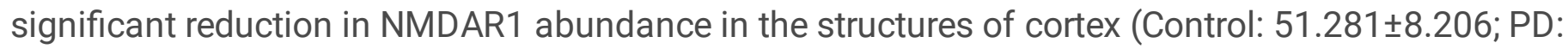

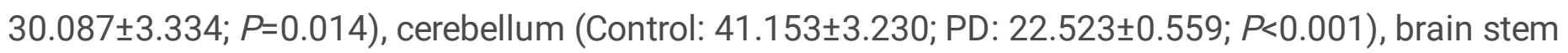
(Control: 51.813 $\pm 6.278 ;$ PD: 24.410 $\pm 3.593 ; P=0.003$ ) and hippocampus (Control: 66.822 \pm 5.785 ; PD: 36.843 \pm 7.843; $P=0.006)$ in the MPTP-administrated groups relative to their controls, with the exception of cerebellum and midbrain (Fig. 1c). No significant difference was detected in midbrain (Control: 36.619 \pm 5.495 ; PD: 37.669 $\pm 3.285 ; P=0.790$ ) between the MPTP-administrated group and its control,

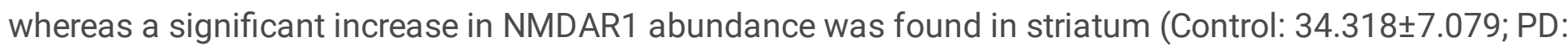


44.750 $\pm 4.142 ; P=0.022)$ in the MPTP-administrated group compared to its control (Fig. 1c). Thus, the results obtained from $\mathrm{IHC}$ were consistent with the ones from Western blot.

Combining the Western blot analysis with IHC examination, it was found that NMDAR1 expression maintained unchangeable in midbrain, highly increased in striatum, and dramatically decreased in cortex, cerebellum, brain stem, and hippocampus in the MPTP-administrated groups compared to their controls.

\section{A tissue-specific distribution of D-serine was found in the mice of MPTP-administrated group}

As abnormally raised D-serine level is associated with NMDAR1-mediated neurotoxicity, we performed an HPLC analysis to evaluate D-serine level in the various specialized structures of mouse brains. In the present study, a fluorescent labeling reagent (OPA/NAC) has been applied to determine D-serine contents. This HPLC method provided a sensitive, efficient analysis of the polar amino acids with trace contents, and was validated by evaluating the linearity of calibration curves for the standard $D$-serine $(r 2>0.9990)$. D-serine concentrations were compared using paired $t$-tests. Data were analyzed using SPSS software package, and comparison results with the level of $p<0.05$ were assigned significance.

The contents of $D$-serine in the various specialized structures of mouse brains were summarized in Table 1. As reported in Table 1, the contents of D-serine were significantly lower in the structures including cortex, cerebellum, brain stem and hippocampus in the mice of MPTP-administrated groups than the ones in the control groups. However, a significantly enhanced D-serine content was revealed in the striatum in the MPTP-administrated mice compared to its control. In addition, there was no significant difference of D-serine content in midbrain between the MPTP-administrated mice and their control mice.

Basically, the distribution of D-Serine was in agreement with NMDAR1 expression in the detected structures of mouse brains. Their changes in these detected structures substantially maintained in the same trends. And they increased in the striatum, and maintained unchangeable in midbrain, but decreased in cortex, hippocampus, cerebellum and brainstem. Base on this finding, it was highly suspected that D-Serine and NMDAR1 were cooperatively distributed and displayed a synergistic effect in mouse brains. And higher D-Serine content/enhanced NMDAR1 expression in striatum, and lower DSerine content/decreased NMDAR1 abundance in cortex, hippocampus, cerebellum and brainstem were suspected to be associated with PD. Additionally, the tissue-specific distribution of D-Serine /NMDAR1 was helpful to elucidate the controversial levels of D-serine in different structures in brains under conditions of the various neural diseases.

Table 1 A tissue-specific analysis of D-serine content via HPLC 


\begin{tabular}{|llll|}
\hline \multirow{2}{*}{ Structures } & \multicolumn{2}{l|}{ D-Serine concentration $(\mathrm{ng} / \mathrm{ml})$} & $P$ \\
\cline { 2 - 3 } & Control & PD & \\
\hline Cortex & $59.274 \pm 4.615$ & $42.417 \pm 6.974$ & $0.025^{\star}$ \\
\hline Cerebellum & $56.950 \pm 6.862$ & $42.198 \pm 3.699$ & $0.031^{\star}$ \\
\hline Brainstem & $57.524 \pm 5.423$ & $45.074 \pm 3.110$ & $0.026^{\star}$ \\
\hline Striatum & $20.087 \pm 4.354$ & $35.580 \pm 4.402$ & $0.012^{\star}$ \\
\hline Midbrain & $45.966 \pm 6.410$ & $49.463 \pm 2.962$ & 0.439 \\
\hline Hippocampus & $66.167 \pm 8.679$ & $23.084 \pm 4.678$ & $0.002^{\star}$ \\
\hline
\end{tabular}

All analyses were conducted in triplicate, and $*, P<0.05$

\section{Establishment of cell model with parkinsonian neurotoxicity using the rat C6 glioma cells}

To investigate the effect of D-serine/NMDAR1 on PD pathogenesis and reveal the relationship between NMDAR1 and D-serine, the rat $\mathrm{C} 6$ glioma cells treated with $\mathrm{MPP}^{+}$was applied to establish a cell model with PD-like neurotoxicity. Since NMDAR1 is secreted by astrocytes in brains, the rat $\mathrm{C} 6$ glioma cells were applied in this study. In addition, cortex is rich in astrocytes, so the results in cortex may provide a reference for assays in the rat $\mathrm{C} 6$ glioma cells.

Morphological changes: MPP ${ }^{+}$, an active form of MPTP in vivo, is worldwide used for preparing cell model with parkinsonian neurotoxicity. The morphological changes of the rat $\mathrm{C} 6$ glioma cells after treatment with $0,1.5,2.0,2.5,5.0$ and $7.5 \mu \mathrm{mol} / \mathrm{L}$ of $\mathrm{MPP}^{+}$were photographed in this study. As shown in Fig. 2a, $\mathrm{MPP}^{+}$displayed obvious neurotoxic effects on the rat $\mathrm{C} 6$ glioma cells in a dose-dependent manner. Shrinking size and conglobated shape were found in the $\mathrm{MPP}^{+}$-treated $\mathrm{C} 6$ cells, whereas regular fusiform shapes were maintained in the untreated cells all the way. With increase of the MPP ${ }^{+}$ concentration, the $\mathrm{C} 6$ cells turned from regular fusiform shapes to conglobated shape gradually in appearance. Till the $\mathrm{MPP}^{+}$concentration was $2.5 \mathrm{mmol} / \mathrm{L}$, the $\mathrm{C} 6$ cells were completely converted into conglobated shapes which may float in the medium (Fig. 2a).

Cell viability assay: To investigate the neurotoxicity of $\mathrm{MPP}^{+}$to the rat $\mathrm{C} 6$ glioma cells, a conventional MTT assay was used to evaluate cell viability in this study. The outcome of MTT assay suggested that the $\mathrm{C} 6$ glioma cells showed a substantial decline in number upon $\mathrm{MPP}^{+}$treatment in a dose-dependent manner (Fig. 2b), and thus revealing its strong neurotoxic effect. Finally, $2 \mathrm{mmol} / \mathrm{ml} \mathrm{MPP}^{+}$was determined to trigger parkinsonian neurotoxicity of the rat $\mathrm{C} 6$ glioma cells as an inducer.

Assay of DA and TH contents: The parkinsonism of the rat $\mathrm{C} 6$ glioma cells treated with $\mathrm{MPP}^{+}$was characterized by measuring the intracellular abundance of TH and concentration of DA. As shown in Fig. $2 \mathrm{c}$ and $2 \mathrm{~d}$, TH expression examined by Western blot analysis displayed a dramatic reduction upon MPP ${ }^{+}$ 
treatment. The comparison analysis on $\mathrm{TH}$ abundance revealed a significant decrease in the $\mathrm{MPP}^{+}-$ treated cells relative to the untreated cells (Fig. 2d). Table 2 and Fig. 2e show that DA content significantly drops to $58.1 \%$ in the MPP ${ }^{+}$-treated cells $(12.237 \pm 1.341)$ compared with the untreated cells $(21.077 \pm 2.191, P=0.004)$.

D-Ser contents and NMDAR1 abundances: Based on the association of D-Serine and NMDAR1 with PD, we analyze the D-Serine content and NMDAR1 abundance in the rat C6 glioma cells. As depicted in Fig. $2 f$, D-serine content was dramatically decreased in the MPP ${ }^{+}$-treated rat C6 glioma cells $(8.121 \pm 1.806)$ compared to the untreated cells $(33.239 \pm 1.869, P<0.01)$. NMDAR1 expression was significantly downregulated in the $\mathrm{MPP}^{+}$-treated cells $(0.355 \pm 0.070)$ relative to the untreated cells $(0.503 \pm 0.089, P=0.019)$ (Fig. $2 \mathrm{~g}$ and $2 \mathrm{~h}$ ).

Consequently, morphological observation, cell viability, DA content and TH abundance analysis together confirmed the successful establishment of the rat $\mathrm{C} 6$ glioma model with a PD-like characterization by exposing the $\mathrm{C} 6$ glioma cells to MPP ${ }^{+}$. Additionally, D-serine content and NMDAR1 expression were found to be decreased in the PD-like cells. Thus, we applied a D-serine supplement to rat $\mathrm{C} 6$ glioma cells further to investigate the effect of $\mathrm{D}$-serine on neurotoxicity of $\mathrm{MPP}^{+}$to the rat $\mathrm{C} 6$ glioma cells.

\section{Protection of D-serine supplement against the MPP+-induced neurotoxicity in the rat $\mathrm{C} 6$ glioma cells}

Design of Groups: To investigate the effect of D-serine on the rat C6 glioma cells with PD-like characterization, we introduced D-serine into the rat C6 glioma cells. Totally, there were 4 groups in the $\mathrm{MPP}^{+}$-treated group, which were 0 (no any intervention reagents), D-Ser40 (40ng/ml D-Serine), D-Ser100 (100ng/ml D-Serine) and Levodopa $(100 \mu \mathrm{mol} / \mathrm{L})$. The untreated rat $\mathrm{C} 6 \mathrm{glioma}$ cells with their corresponding modulators were used as the controls in this study.

Morphology: Morphological observation revealed that D-Serine played a role in maintaining regular size and adherent growth of the rat $\mathrm{C} 6$ glioma cells. Compared with the cells grown under no intervention reagents after $\mathrm{MPP}^{+}$exposure, the $\mathrm{MPP}^{+}$-treated rat $\mathrm{C} 6$ glioma cells grown under $\mathrm{D}$-serine showed a decrease in numbers of the suspended cells with conglobated shape, and most cells apparently were under normally adherent growth (Fig. S1).

DA content: To investigate whether D-Serine played a role in attenuating MPP ${ }^{+}$-induced neurotoxicity, the typical parameters of parkinsonism including TH, Ach and DA were analyzed in this study. DA content was evaluated using HPLC. As presented in Table 2, pretreatment with $\mathrm{MPP}^{+}$led to DA content to be significantly declined in the rat $\mathrm{C} 6$ glioma cells. When D-Serine was added into the MPP ${ }^{+}$-treated rat $\mathrm{C} 6$ glioma cells, the DA content was slightly increased and stably maintained at a relatively low level. However, for the untreated rat C6 glioma cells grown under D-Serine, a low level of DA was detected as well, and no significant difference in DA content was found between the MPP ${ }^{+}$-treated $(14.902 \pm 0.369)$ and untreated rat $\mathrm{C} 6$ glioma cells $(16.244 \pm 2.923 ; P=0.474)$ grown under $100 \mathrm{ng} / \mathrm{ml} \mathrm{D}$-Serine. Thereby, this 
evidence suggested that D-Serine displayed an activity to maintain DA content at a relatively low level whatever the cells were exposed to $\mathrm{MPP}^{+}$or not.

TH expression: TH expression was evaluated in various cellular combinations using Western blot analysis. As depicted in Fig. 3a and 3c, when D-Serine was added into the MPP ${ }^{+}$-treated rat C6 glioma cells, the TH expression was raised (D-Ser40, 0.324 $\pm 0.014, P<0.001$; D-Ser100, 0.327 $\pm 0.013, P<0.001$ ) compared with their 0 group $(0.174 \pm 0.005)$. In addition, a significant increase in TH expression was found in the D-Serine-modulated groupsin the $\mathrm{MPP}^{+}$-treated cells compared to the untreated rat $\mathrm{C} 6$ glioma cells (D-Ser40: Control, $0.195 \pm 0.008 ; P D, 0.324 \pm 0.014 ; P<0.001$. D-Ser100: Control, $0.238 \pm 0.019$; $\mathrm{PD}, 0.327 \pm 0.013 ; P=0.003)$. Thereby, TH expression analyzed by Western blot was in agreement with the DA content detected in this study. As a whole, D-Serine was found to have a role in maintaining $\mathrm{TH}$ at a relatively low level in the MPP ${ }^{+}$-treated/untreated groups, but higher than the ones in the MPP ${ }^{+}$-treated cells without any modulators.

ACh content: DA and ACh are the two main neuromodulators in CNS (central nervous system), and the unbalance of DA/ACh in striatum leads to the motor dysfunction of a patient with PD. Usually, a reduction in DA and hyperfunction of ACh were found in PD patients. Therefore, currently effective treatments target the DA/ACh modulatory systems $[1,27,28]$. Based on the importance of the DA/ACh balance, ACh contents in the rat $\mathrm{C} 6$ glioma cells were evaluated using commercially available ACh quantitation kit. As shown in Table 2, unlike dramatic increase in ACh content in the 0 group (Control, 14.920 1.315 ; PD, 42.998 $\pm 2.562 ; P<0.001)$, no significant difference was found in the $D$-Serine-modulated groups between the MPP ${ }^{+}$-treated and untreated rat glioma cells. In addition, we found that D-Serine showed a strong activity to reduce ACh contents to the normal level in the MPP+-treated rat glioma cells, further maintaining ACh contents similar to the levels in their corresponding untreated cells. Although D-serine maintained the level of DA and ACh at a low level, it remained the balance of DA/ACh constant (D-ser100: Control, 1.131; PD, 1.121) compared to the control groups (0: Control, 1.413; PD, 0.258). Thus, it was proposed that $\mathrm{D}$-serine play a role in maintaining the $\mathrm{DA} / \mathrm{ACh}$ balance stable.

Thus, D-Serine supplement shows a role in maintaining intracellular TH increased, further attenuating the pathogenic changes in PD-like cell models. Thereby, D-Serine strongly displays a role in recuing cells from $\mathrm{MPP}^{+}$-induced neurotoxicity.

Table 2 Statistic analysis of representative parkinsonian parameters and apoptosis-related factors in the rat C6 glioma cells 


\begin{tabular}{|c|c|c|c|c|c|}
\hline \multirow[t]{2}{*}{ DA } & Control & \multicolumn{2}{|l|}{$\mathrm{PD}$} & \multirow[t]{2}{*}{$P$} & \\
\hline & Mean $\pm S D$ & \multicolumn{2}{|c|}{ Mean $\pm S D$} & & \\
\hline 0 & $21.077 \pm 2.191$ & \multicolumn{2}{|c|}{$12.237 \pm 1.341$} & \multicolumn{2}{|c|}{$0.004 *$} \\
\hline D-Ser40 & $18.451 \pm 1.195$ & \multicolumn{2}{|c|}{$14.726 \pm 2.028$} & \multicolumn{2}{|c|}{0.052} \\
\hline D-Ser100 & $16.244 \pm 2.923$ & \multicolumn{2}{|c|}{$14.902 \pm 0.369$} & \multicolumn{2}{|c|}{0.474} \\
\hline Levodopa & $15.906 \pm 0.726$ & \multicolumn{2}{|c|}{$13.000 \pm 1.274$} & \multicolumn{2}{|c|}{$0.027 *$} \\
\hline \multirow[t]{2}{*}{ ACh } & Control & \multicolumn{3}{|c|}{ PD } & \multirow[t]{2}{*}{$P$} \\
\hline & Mean $\pm S D$ & $P($ to 0$)$ & Mean $\pm S D$ & $P$ (to 0 ) & \\
\hline 0 & $14.920 \pm 1.315$ & & $42.998 \pm 2.562$ & & $<0.001^{\star}$ \\
\hline D-Ser40 & $11.328 \pm 1.646$ & $0.042^{\star}$ & $11.604 \pm 0.548$ & $<0.001^{\star}$ & 0.796 \\
\hline D-Ser100 & $14.367 \pm 4.066$ & 0.834 & $13.296 \pm 1.352$ & $<0.001^{*}$ & 0.688 \\
\hline Levodopa & $40.768 \pm 5.377$ & $0.001^{\star}$ & $36.275 \pm 6.387$ & 0.166 & 0.404 \\
\hline \multirow[t]{2}{*}{ Calcium } & Control & \multicolumn{3}{|c|}{ PD } & \multirow[t]{2}{*}{$P$} \\
\hline & Mean $\pm S D$ & $P($ to 0$)$ & Mean $\pm S D$ & $P($ to 0$)$ & \\
\hline 0 & $3.757 \pm 0.173$ & & $5.142 \pm 0.519$ & & 0.012 \\
\hline D-Ser40 & $3.751 \pm 0.449$ & 0.985 & $4.312 \pm 0.171$ & 0.058 & 0.113 \\
\hline D-Ser100 & $3.833 \pm 0.235$ & 0.675 & $3.552 \pm 0.500$ & $0.019 *$ & 0.429 \\
\hline Levodopa & $3.154 \pm 0.370$ & 0.063 & $4.252 \pm 0.369$ & 0.073 & 0.022 \\
\hline \multirow[t]{2}{*}{ ROS } & Control & \multicolumn{3}{|c|}{ PD } & \multirow[t]{2}{*}{$P$} \\
\hline & Mean $\pm S D$ & $P($ to 0$)$ & Mean $\pm S D$ & $P($ to 0$)$ & \\
\hline 0 & $11.989 \pm 0.841$ & & $14.720 \pm 0.490$ & & $0.001^{\star}$ \\
\hline D-Ser40 & $12.231 \pm 0.328$ & 0.610 & $14.137 \pm 0.907$ & 0.302 & $0.008^{*}$ \\
\hline D-Ser100 & $11.949 \pm 0.475$ & 0.937 & $12.856 \pm 1.050$ & $0.018^{*}$ & 0.167 \\
\hline Levodopa & $8.791 \pm 0.631$ & $<0.001 *$ & $9.069 \pm 0.846$ & $<0.001^{\star}$ & 0.617 \\
\hline
\end{tabular}

DA level was assessed by HPLC, and the contents of ACh, ROS and calcium in the various cellular combinations were analyzed using ELISA (enzyme-linked-immunosorbent assay). All analyses were conducted in triplicate, and $*, P<0.05$. $P$ (to 0$)$, comparison occurred between 0 group and other groups with various modulators in the MPP ${ }^{+}$-treated or untreated group; $P$, comparison occurred between $\mathrm{MPP}^{+}$ treated cells and their corresponding untreated cells. 


\section{D-Serine attenuated cell death in the MPP+-treated rat glioma cells}

To assess the mechanism underlying the effect of D-Serine supplement on the MPP ${ }^{+}$-induced neurotoxicity and cell apoptosis, AnnexinV-FITC/PI staining and flow cytometry were applied in this study, and the apoptosis of rat glioma cells was analyzed using cell percentages.

As shown in Fig. 3e, the rate of apoptosis and death for the rat glioma cells maintained dynamically stable in all control groups with the exception of the Levodopa group. Fig. 3e clearly shows that the dead cells dramatically increase from $4.39 \%$ to $23.5 \%$ and late apoptotic cells raise from $2.45 \%$ to $8.02 \%$ in Levodopa group compared to 0 group. Thus, it showed that Levodopa played a role in dramatically inducing cell death. For the $\mathrm{MPP}^{+}$-treated group, $\mathrm{MPP}^{+}$exposure significantly raised the rate of apoptosis and death for the rat glioma cells compared to the untreated cells. In addition, Fig. $3 e$ displays that the dead cells drop from $12.6 \%$ in $\mathrm{MPP}^{+} / 0$ group to $11.9 \%$ in MPP+/D-Ser100 group, and the early apoptotic cells decrease from $7.93 \%$ in $\mathrm{MPP}^{+} / 0$ group to $5.19 \%$ in MPP+/D-Ser100 group. Thereby, it was found that the pretreated cells in the groups of D-serine-modulated group displayed a potential to attenuate the $\mathrm{MPP}^{+}$-induced cytotoxicity of the rat glioma cells. Thus, D-Serine supplement showed a role in decreasing cell death for the MPP+-treated cells. These results were confirmed by Hoechast staining as well (Fig. S2).

Therefore, it was found that D-Serine showed an activity to attenuate the cell apoptosis and death, whereas Levodopa displayed an opposite role in modulating cell apoptosis and death, and delivered a neurotoxicity to the rat $\mathrm{C} 6$ glioma cells. Potentially, this result may be helpful to elucidate the progressive side-effects occurred by Levodopa. Therefore, the percentage of apoptotic cells and dead cells was dramatically decreased in D-Serine-modulated groups pretreated with $\mathrm{MPP}^{+}$. Thereby, D-Serine therapies protected the cells from MPP+-induced neurotoxicity and death.

\section{D-Serine attenuated cell death via inhibiting calcium-DAPK1 related transduction pathway}

Apoptosis is a programmed cell death, which is regulated by either anti-apoptotic factors or pro-apoptotic factors. Bcl-2 is a major factor in the family of anti-apoptotic factors, and DAPK1 and its relative molecules in the same signal transduction pathway are in the class of pro-apoptotic factors. In this study, the above parameters were investigated to clarify the possible signal transduction pathway related to $D$ Serine.

D-Serine enhanced NMDAR1 expression: Based on the evidences that NMDAR1 were cooperatively distributed with D-Serine in mouse brains, we examined the NMDAR1 abundance in D-Serine-modulated C6 cells with pretreatment of MPP ${ }^{+}$via ICC (Fig. 3c) and Western blot (Fig. 3a), and the D-Serinemodulated $\mathrm{C} 6$ cells without pretreatment of $\mathrm{MPP}^{+}$was used as the controls in this study. As shown in Fig. $3 \mathrm{~b}$ and $3 \mathrm{~d}$, the statistic analysis on NMDAR1 expression in ICC images was consistent with the one in the Western blot analysis. Treatment with D-Serine significantly up-regulated the expression of NMDAR1 in 
the $\mathrm{C} 6$ cells compared to their corresponding controls (Fig. 3b). Therefore, D-serine displayed a strong activity to promote NMDAR1 expression at cellular level.

D-Serine enhanced Bcl2 expression: To examine whether D-Serine treatment influenced protein expression of anti-apoptotic factors associated with cell apoptosis, we performed a Western blot for analyzing Bcl2 expression in D-Serine-modulated C6 cells (Fig. 3a). Densitometric analysis indicated that D-Serine induced a significant increase in Bcl2 expression, whereas Levodopa did not display an activity to change the $\mathrm{Bcl} 2$ expression (Fig. 3d). Thus, it was found that D-serine promoted intracellular Bcl2 expression.

D-Serine inhibited DAPK1 expression: To assess whether D-Serine treatment influenced protein expression of pro-apoptotic factors associated with cell apoptosis, and elucidate the potential signal transduction pathway involved, we examined DAPK1 expression in D-Serine-modulated C6 cells using Western blot (Fig. 3a). As shown in Fig. 3d, D-Serine and Levodopa had a strong activity to down-regulate DAPK1 expression. Thus, D-serine played a role in attenuating cellular DAPK1 expression.

D-Serine maintained calcium content in low level in the MPP+-induced cells: Due to a significant correlation of NMDAR1 and DAPK1 with calcium, an intracellular calcium level was examined using the fluorescent probe Fluo-3AM as shown in Table 2. Spectrophotometric analysis revealed that the level of intracellular calcium remained stable in the control populations (Table 2). However, it was significantly higher in the MPP ${ }^{+}$-pretreated $\mathrm{C} 6$ cells $(5.142 \pm 0.519)$ compared with the un-pretreated cells $(3.757 \pm 0.173$, $P=0.012)$ without any modulators. Assay of $\left[\mathrm{Ca}^{2+}\right]_{\mathrm{cyt}}$ showed a dramatic reducing trend in the $\mathrm{MPP}^{+}-$ pretreated $\mathrm{C} 6$ cells with exposure of D-serine (Table 2). Thus, D-serine showed an activity in efficiently decreasing intracellular calcium level in the MPP+ ${ }^{+}$-pretreated $\mathrm{C} 6$ cells.

D-Serine reduced ROS contents: Based on causative influence of ROS production on PD, we investigated ROS levels in the rat $\mathrm{C} 6$ glioma cells. As shown in Table 2, compared with 0 group, Levodopa showed a strong activity to decrease ROS level in both MPP+-pretreated (0: 14.720 \pm 0.490 ; Levodopa: $9.069 \pm 0.846$; $P<0.001)$ and untreated C6 cells (0: 11.989 \pm 0.841 ; Levodopa: 8.791 $\pm 0.631 ; P<0.001)$. D-Serine at the dose of $100 \mathrm{ng} / \mathrm{ml}$ was found to significantly decrease ROS in the MPP ${ }^{+}$-pretreated C6 cells (0: 14.720 $\pm 0.490 ; D-S e r 100: 12.856 \pm 1.050 ; P=0.018$ ). Additionally, there were no difference in RoS level that were found in the D-serine-modulated groups at the dose of $100 \mathrm{ng} / \mathrm{ml}$ between the MPP ${ }^{+}$-treated cells and untreated cells, whereas a significant increase in ROS level was found in 0 group (Control, $11.989 \pm 0.841 ; P D, 14.720 \pm 0.490 ; P=0.001$ ) and $D-S e r 40$ group (Control, $12.231 \pm 0.328 ; P D, 14.137 \pm 0.907$; $P=0.008$ ) in the $\mathrm{MPP}^{+}$-treated cells compared to the untreated cells. Thereby, these results suggested that D-Serine with high concentration attenuated oxidative damage induced by MPP'.

\section{D-Serine treatment ameliorated the parkinsonian symptoms in the MPTP-administrated mice}

MPTP is worldwide used to prepare mouse model with PD because it can reproduce the similar essential symptoms in mice to the ones in parkinsonian patients. In this study, a successful mouse model with PD was constructed by intraperitoneal injection of MPTP and confirmed by detecting the reduction in DA and 
$\mathrm{TH}$, and examining motor dysfunction[26]. Then, the MPTP-administrated mice were treated with D-serine, and their motor functions and representative parkinsonian parameters including striatal DA and mesencephalic TH were measured. The MPTP-administrated mice were injected with physiological saline and Levodopa/Benzylhydrazine were used as controls.

D-Serine treatment slightly ameliorated the impairment of motor function: To assess whether the D-Serine treatment ameliorated the motor impairment of the MPTP-administrated mice, a locomotor activity was evaluated between the D-serine-treated mice and their controls. As depicted in Fig. 4a, although the impairment of motor function for the mice wasn't completely recovered from the MPTP injury in the MPTP/D-serine group, the D-serine treatment displayed an ability to ameliorate the locomotor impairment to a certain extent compared to the healthy and MPTP-administrated mice. The horizontal grid test, which allowed the assessment of the forepaw faults, showed a significant increase in hanging duration for the mice in the MPTP/D-serine group compared to the ones in the MPTP group as shown in Fig. 4a. The vertical grid test, which illustrated the assessment of the fore-paw strength, displayed a reduction in latency of climbing pole for mice in the MPTP/D-serine group relative to the ones in the MPTP group (Fig. 4a). Thereby, although D-serine cannot reverse the brain damages induced by MPTP, D-serine treatment was found to be beneficial to the amelioration of motor dysfunction from the MPTP injury.

An increase in striatal DA, mesencephalic TH and cortical NMDAR1 was found in D-serine-treated mice: In support of behavioral tests, we conducted an HPLC analysis on striatal DA, and a Western blot analysis on mesencephalic TH and cortical NMDAR1. HPLC analysis revealed an increase in DA content in the mice of the MPTP/D-serine group compared to the MPTP group as illustrated in Fig. 4b. TH detection and its statistic analysis displayed an enhanced expression in TH protein in midbrain of the mice in the MPTP/D-serine group relative to the MPTP group (Fig. 4c and 4d). In addition, cortical NMDAR1 was tested in this study. Fig. $4 \mathrm{c}$ and $4 \mathrm{~d}$ showed that D-serine injection increase NMDAR1 abundance in cortex of the mice in the MPTP/D-serine group compared to the MPTP group. Thus, we found that D-serine treatment showed a potential to enhance the TH expression in midbrain, raise the DA content in striatum, and increase the NMDAR1 abundance in cortex. Those evidences were consistent with the ones obtained at the cellular level.

\section{Discussion}

Elevated levels of D-serine/NMDAR1 were highly suspected to be involved in cell rescues under PD conditions

Our study showed a tissue-specific distribution of D-Serine and NMDAR1. We found that the content of Dserine was consistent with the abundance of NMDAR1 in the various structures of mouse brains, and an increase in striatum, no significant changes in midbrain, a decrease in cortex, hippocampus, cerebellum and brainstem for D-serine content and NMDAR1 abundance were revealed in mouse brains. It is well known that there are four major dopaminergic pathways in brains including the nigrostriatal pathway/mesostriatal pathway, mesocortical pathway, mesolimbic pathway and tuberoinfundibular 
pathway. Among these, the nigrostriatal pathway initiates from SNpc in midbrain and ends in striatum, and it particularly plays an important role in regulating cognition, motor behavior, and sleep-wake states $[29,30]$. Nigrostriatal pathway is the efferent connection of DA between the SNpc where DA synthesizes and striatum. A marked reduction in dopamine function in nigrostriatal pathway is most pronounced pathological features of PD. Therefore, Parkinson's disease is characterized by degeneration of the nigrostriatal dopaminergic system, resulting in the motor features of the disease [31]. Based on our study, since D-serine level varied throughout the brain, it appeared likely that the relative contribution of Dserine to NMDAR1 activation was heterogeneous in various structures of the MPTP-administrated mouse brain. And a dramatic increase in D-serine content and NMDAR1 expression in nigrostriatal dopaminergic system was revealed, whereas a significant reduction was found in the other four structures including cortex, hippocampus, cerebellum and brainstem. Maybe, that's why there are controversial reports on Dserine levels in the various structures of brains for AD patients [20]. NMDAR1 is reported to be secreted by astrocyte which is universal in cortex, and a reduced D-serine/NMDAR1 levels were found in cortex.

Therefore, it is conceivable that a reduction in cortical D-serine levels could be involved in brain disorders in which NMDARs contributes to the pathophysiology of PD, and it is overwhelmingly likely that a patient with PD has got a symptom of cognitive dysfunction, motor impairment, anxiety and depression, etc. In addition, a synergistic effect of D-serine and NMDAR1 was found, and an elevated D-Serine level was revealed in nigrostriatal dopaminergic system in this study. And D-serine supplement was found to protect neuronal cells against the $\mathrm{MPP}^{+}$-induced neurotoxicity. Thus, we hypothesized that an increase in D-serine/NMDAR1 may result in cell rescues in the nigrostriatal dopaminergic system of mouse brains, and up-regulated D-serine/NMDAR1 may promote cells in nigrostriatal dopaminergic system to survive under PD conditions.

\section{Active sites of NMDAR1 bound to D-serine/glycine was evaluated by molecular docking using MOE software package}

In this study, bioinformation of NMDAR1 was evaluated. The sequence of NMDAR1 was retrieved from https://www.ncbi.nlm.nih.gov/, and accession number is Q05586. NMDAR1 is a 938-aa-long protein containing 3 transmembrane regions at $\mathrm{C}$ terminus. NMDAR1 was highly homologous between homo sapient and rattus norvegicus, an amino acid sequence with $99.25 \%$ identity and $99.79 \%$ similarity between these two species was found. In addition, an effort was made to access the active sites of NMDAR1 interacted with D-serine/glycine by means of MOE (Molecular Operating Environment) software package in this study. NMDAR1 structure was obtained from Protein Data Bank [PDB Code1PB8]. NMDAR1 in the file of 1PB8 was composed of 292 amino acids. It started at Met394 and ended with Ser800 of the native NR1 sequence. In addition, a GT linker replaced the transmembrane regions between Met394 and Ser800 at original sequence of NMDAR1 [32]. MOE evaluation on 1PB8 illustrated the binding site of glycine/D-serine with NMDAR1 as shown in Fig. 5a. Based on the model of glycine interacted with NMDAR1, we found that Arg131 interacted with the two oxygen atoms at the carboxyl group of glycine, Thr126 and Leu125 attacked oxygen doubly bonded to carbon at the carboxyl group, and Ser180 nucleophilically attacked oxygen at hydroxyl group of $\mathrm{COOH}$ and was electrophilically 
attacked by central carbon of glycine. Additionally, $\mathrm{NH}_{3}{ }^{+}$interacted with Pro124, Asp224, and Thr126 of NMDAR1 by an electrophilic attack. Mostly, the atoms which interacted with NMDAR1 in the model of Dserine bound protein were similar to the ones in the model of glycine-bound NMDAR1. The major deference depends on the functional $\mathrm{CHOH}$ group. The oxygen of hydroxyl group was electrophilically attacked by Ser180 and Phe92. Thereby, Arg131, Thr126, Ser180, Asp224, Leu125, Phe 92 and Pro124 were all active site for the binding of D-serine/glycine with NMDAR1. In this study, we elucidated the 3D structure of NMDAR1 retrieved from PDB database and active sites of NMDAR1 interacted with Dserine/glycine. This work recognized the specific active sites of NMDAR1 bound to D-serine/glycine, which enabled us to predict the possible structurally similar ligands to D-serine necessary for the NMDAR1 activity, or design D-serine derivatives based on the active center pockets and ligand interaction assays. Thus, MOE prediction provides a structural framework for drug designs targeted NMDAR1 against PD.

\section{D-Serine attenuated neuronal cell death via DAPK1-related pathway}

To further unravel the effect of D-serine/NMDAR on neuronal cells, rat C6 glioma cells were introduced in this study. Based on the finding that NMDAR1 was synergistically altered with D-serine level, the NMDAR1 abundance was analyzed using the rat $\mathrm{C} 6$ glioma cells by introducing D-serine in. As a consequence, we found that D-Serine augmentation significantly up-regulated NMDAR1 expression whatever at low $(40 \mathrm{ng} / \mathrm{ml})$ or high concentration $(100 \mathrm{ng} / \mathrm{ml})$. Thus, we speculated that D-serine displayed a strong activity in facilitating NMDAR1 expression at cellular level.

NMDAR is an ionotropic channel of cations $\left(\mathrm{Na}^{+}, \mathrm{K}^{+}, \mathrm{Ca}^{2+}\right)$, and its opening participates in regulating calcium efflux. NMDAR exhibits high permeability to calcium. When NMDAR is activated, a slow $\mathrm{Ca}^{2+}$ permeable component of the excitatory postsynaptic currents (EPSCs) will be produced. Due to close relationship of NMDAR1 with calcium, the level of intracellular $\mathrm{Ca}^{2+}$ was tested in this study. As a consequence, a dramatic reduction in $\left[\mathrm{Ca}^{2+}\right]_{\mathrm{cyt}}$ was found when the $\mathrm{MPP}^{+}$-pretreated $\mathrm{C} 6$ cells was exposed to D-serine. It is reported that D-serine, as an agonist of NMDAR, plays a role in facilitating

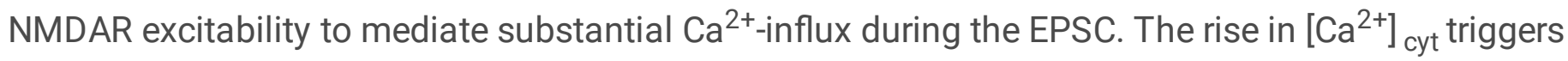
downstream multiple changes in the postsynaptic neuron as a signal, such as the changes in synaptic efficacy and neuronal morphology which cause either short-term or long-term effects [33, 34]. However, the results in our study were contrary to that. Our study illustrated that D-serine decreased the concentration of intracellular calcium ion. Based on our study, we bravely hypothesized that D-serine didn't display an activity to excite NMDAR1, but inhibit NMDAR1. Like a competitive inhibitor with structural similarity, when D-serine bound to the active center of NMDAR1, glycine was unable to activate NMDAR1 since they bound with NMDAR1 at the same active site. In addition, NMDAR1 expression was increased by $\mathrm{D}$-serine in the $\mathrm{MPP}^{+}$-pretreated $\mathrm{C} 6$ cells based on a compensatory mechanism in response to the inhibition of NMDAR activity. D-serine/NMDAR1 displayed a potential to inhibit cells to be overactivated. It was beneficial to explain why the D-serine/NMDAR1 was enhanced in the nigrostriatal dopaminergic system. 
It is well established that DAPK1 causes neuronal cell death via NMDAR under pathological conditions [35]. DAPK1 increases the flux of $\mathrm{Ca}^{2+}$ ions through NMDAR by DAPK1-dependent phosphorylation of Ser1303 at the C-terminal region of GluN2B [36, 37]. Due to the close association of DAPK1 with NMDAR and calcium, the abundance of DAPK1 was assessed under the conditions with Dserine supplement. As a consequence, we found the D-serine supplement caused a decreased DAPK1 expression. DAPK1 is a calcium/calmodulin (CaM)-regulated Ser/Thr kinase with a molecular weight of $160 \mathrm{kDa}$. Its activity is regulated by $\mathrm{Ca}^{2+} / \mathrm{CaM}$. The CaM activated by $\mathrm{Ca}^{2+}$ enhances the catalytic activity of DAPK1 by phosphorylating Ser308 of DAPK1[38]. DAPK1 plays a critical role in neuronal cell death. For example, it induces synucleinopathy and degeneration of dopaminergic neurons in PD. The activation of DAPK1 directly leads to apoptotic cell death via a various forms including TNF-a-, Fas-, TGF- $\beta$-, ceramide-, caspase-, amyloid-beta (AB)-, and p53-mediated apoptosis [35]. In our study, a reduced intracellular $\mathrm{Ca}^{2+}$ and DAPKI were detected. Thereby, these evidences suggested that supplement of D-serine decreased cytosolic $\mathrm{Ca}^{2+}$ through inhibiting NMDAR activity and attenuated neuronal cell death via inhibiting DAPK1-associated apoptotic pathway as shown in Fig. 5b. Additionally, the recuing role of Dserine supplement in cell death and apoptosis was further demonstrated by the following evidences including the raised $\mathrm{Bc} 22$ expression, the photographed cell morphology, and the decreased ROS content in the cell models. Therefore, we proposed a hypothesis that D-Serine attenuated neuronal damage by inhibiting NMDAR1 activity, and inhibited DAPK1-associated signaling pathways which mediated by $\mathrm{Ca}^{2+}$ (Fig. 5b).

\section{Conclusion}

Overall, the tissue-specific D-serine synergistically acted on NMDAR1 was supported to be associated with PD in our finding. In addition, D-serine supplement was found to promote the survival of neuronal cells via promoting NMDAR expression and attenuating DAPK1-associated pathway. Our findings highlight D-serine as a candidate for PD therapy. Unlike the previous study, even at high concentration, Dserine still displayed a protective effect on neuronal cells. This opens up an innovative perspective for neurobiological therapy using D-serine augmentation.

\section{Abbreviations}

NMDAR1: N-methyl-D-aspartate receptor 1; PD: Parkinson's disease; MPTP: 1-Methyl-4-phenyl-1,2,3,6tetrahydropyridine; MPP+: 1-Methyl-4-phenylpyridinium iodide; SNpc: substantia nigra pars compacta; HPLC: high performance liquid chromatography; SDS-PAGE: SDS-acrylamide gel electrophoresis; ECL: an enhanced chemiluminescent; IHC: immunohistochemical; ICC: immunocytochemistry; DA: dopamine; TH: Tyrosine hydroxylase; ACh : Acetylcholine; ROS: reactive oxidative species; GWAS: a genome-wide association study; SR: serine racemase; AD: Alzheimer's disease; DMEM: Dulbecco's modified Eagle's medium; FBS: fetal bovine serum; OPA: o-phthaldialdehyde; NAC: N-acetyl-L-cysteine; PBS: phosphate- 
buffered saline; FITC: fluorescein isothiocyante; PI: propidium iodide; DCFH-DA: 2',7'-dichlorofluorescin diacetate; DCF: fluorescent dichlorofluorscein.

\section{Declarations}

\section{Acknowledgements}

Not applicable.

\section{Authors' contributions}

WZ conceived the study, supervised overall process and composed the manuscript. XS, JL and YP performed the experiments. $Y X$ and $Y Q$ provided the technical supporting. $\mathrm{HZ}$ contributed to data analysis, and WD and LM provided the financial supporting. SL processed the data and modified this manuscript. All authors shared in responsibility for the final decision to submit it for publication.

\section{Funding}

This work was supported in part by National Natural Science Foundation of China (81200989, 81272429), Natural Science Foundation of Liaoning (2019-MS-088), Innovation Team Foundation of Liaoning (LT2010026), and Liaoning Provincial Program for Top Discipline of Basic Medical Sciences.

\section{Availability of data and materials}

The datasets used and/or analysed during the current study are available from the corresponding author on reasonable request.

\section{Ethics approval and consent to participate}

This study was carried out in accordance with the principles of the Basel Declaration and recommendations of Dalian Medical University for laboratory animals. The protocol was approved by the Animal Ethics Committee of Dalian Medical University.

\section{Consent for publication}

Not applicable.

\section{Competing interests}

The authors declare that they have no competing interests.

\section{Author details}

${ }^{1}$ Biochemistry and Molecular Biology Department of College of Basic Medical Sciences, Dalian Medical University, Dalian 116044, China; 
${ }^{2}$ Dalian Sixth People's Hospital, Dalian 116033, China;

${ }^{3}$ Department of Epidemiology, Dalian Medical University, Dalian 116044, China;

${ }^{4}$ Department of Parasitology, Dalian Medical University, Dalian 116044, China;

${ }^{5}$ Department of pharmacochemistry, Dalian Medical University, Dalian 116044, China;

\section{References}

1. Rizzi G, Tan KR. Dopamine and Acetylcholine, a Circuit Point of View in Parkinson's Disease. Frontiers in Neural Circuits. 2017; 11.

2. Grimes D, Fitzpatrick M, Gordon J, Miyasaki J, Fon EA, Schlossmacher M, Suchowersky O, Rajput A, Lafontaine AL, Mestre T, et al. Canadian guideline for Parkinson disease. CMAJ. 2019;191(36):E9891004.

3. Michel PP, Hirsch EC, Hunot S. Understanding Dopaminergic Cell Death Pathways in Parkinson Disease. Neuron. 2016;90:675-91.

4. Anwar A, Saleem S, Akhtar A, Ashraf S, Ahmed MF. Juvenile Parkinson Disease Cureus. 2019;11:e5409.

5. Collaborators GPsD. Global, regional, and national burden of Parkinson's disease, 1990-2016: a systematic analysis for the Global Burden of Disease Study 2016. Lancet Neurol. 2018;17(11):93953.

6. Gazewood JD, Richards DR, Clebak K. Parkinson disease: an update. Am Fam Physician. 2013;87(4):267-73.

7. Edwards TL, Scott WK, Almonte C, Burt A, Powell EH, Beecham GW, Wang L, Zuchner S, Konidari I, Wang G, et al. Genome-wide association study confirms SNPs in SNCA and the MAPT region as common risk factors for Parkinson disease. Ann Hum Genet. 2010;74:97-109.

8. Huang Q, Xie Y, Hu Z, Tang X. Anti-N-methyl-D-aspartate receptor encephalitis: A review of pathogenic mechanisms, treatment, prognosis. Brain Res. 2020;1727:146549.

9. Kaplan E. ASCT1 (SIc1a4) transporter is a physiologic regulator of brain D-serine and neurodevelopment. PNAS. 2018;115:9628-33.

10. Burgdorf JS, Vitaterna MH, Olker CJ, Song EJ, Christian EP, Sorensen L, Turek FW, Madsen TM, Khan MA, Kroes RA, Moskal JR. NMDAR activation regulates the daily rhythms of sleep and mood. Sleep. 2019; 42.

11. Ding H, Jian Z, Stary CM, Yi W, Xiong X. Molecular Pathogenesis of Anti-NMDAR Encephalitis. Biomed Res Int. 2015; 2015:643409.

12. Lee G, Zhou Y. NMDAR Hypofunction Animal Models of Schizophrenia. Front Mol Neurosci. 2019;12:185. 
13. Montes de Oca Balderas P. Flux-Independent NMDAR, Signaling. Molecular Mediators, Cellular Functions, and Complexities. Int J Mol Sci. 2018; 19.

14. Zeng Q, Michael IP, Zhang P, Saghafinia S, Knott G, Jiao W, McCabe BD, Galvan JA, Robinson HPC, Zlobec l, et al. Synaptic proximity enables NMDAR signalling to promote brain metastasis. Nature. 2019;573:526-31.

15. Wang Q, Lv Y, Pang J, Li X, Lu X, Wang X, Hu X, Nie T, Yang X, Xiong YQ, et al. In vitro and in vivo activity of $d$-serine in combination with $\beta$-lactam antibiotics against methicillin-resistant Staphylococcus aureus. Acta Pharmaceutica Sinica B. 2019;9:496-504.

16. Neame S, Safory H, Radzishevsky I, Touitou A, Marchesani F, Marchetti M, Kellner S, Berlin S, Foltyn VN, Engelender $S$, et al. The NMDA receptor activation by d-serine and glycine is controlled by an astrocytic Phgdh-dependent serine shuttle. Proc Natl Acad Sci U S A. 2019;116(41):20736-42.

17. Beltrán-Castillo S, Eugenín J, von Bernhardi R. Impact of Aging in Microglia-Mediated D-Serine Balance in the CNS. Mediators of Inflammation. 2018; 2018:1-11.

18. Henneberger C, Bard L, Rusakov DA. d-Serine: A key to synaptic plasticity? The International Journal of Biochemistry Cell Biology. 2012;44:587-90.

19. Robin LM, Oliveira da Cruz JF, Langlais VC, Martin-Fernandez M, Metna-Laurent M, Busquets-Garcia A, Bellocchio L, Soria-Gomez E, Papouin T, Varilh M, et al. Astroglial CB1 Receptors Determine Synaptic D-Serine Availability to Enable Recognition Memory. Neuron. 2018;98:935-44.e935.

20. Madeira C, Lourenco MV, Vargas-Lopes C, Suemoto CK, Brandao CO, Reis T, Leite RE, Laks J, JacobFilho W, Pasqualucci CA, et al. d-serine levels in Alzheimer's disease: implications for novel biomarker development. Transl Psychiatry. 2015;5:e561.

21. Olney JW, Newcomer JW, Farber NB. NMDA receptor hypofunction model of schizophrenia. J Psychiatr Res. 1999;33:523-33.

22. Ma L, Song J, Sun X, Ding W, Fan K, Qi M, Xu Y, Zhang W. Role of microtubule-associated protein 6 glycosylated with Gal-( $\beta-1,3)-G a l N A c$ in Parkinson's disease. Aging. 2019;11(13):4597-610.

23. Zhang W, Li S, Ma L, Ding W, Xu Y. Identification of a novel carboxypeptidase encoded by Rv3627c that plays a potential role in mycobacteria morphology and cell division. Enzyme Microb Technol. 2019;126:32-40.

24. Tillerson JL, Miller GW. Grid performance test to measure behavioral impairment in the MPTPtreated-mouse model of parkinsonism. J Neurosci Methods. 2003;123:189-200.

25. Kim ST, Son HJ, Choi JH, Ji IJ, Hwang O. Vertical grid test and modified horizontal grid test are sensitive methods for evaluating motor dysfunctions in the MPTP mouse model of Parkinson's disease. Brain Res. 2010;1306:176-83.

26. Ding W, Qi M, Ma L, Xu X, Chen Y, Zhang W. ADP/ATP translocase 1 protects against an alphasynuclein-associated neuronal cell damage in Parkinson's disease model. Cell Biosci. 2021;11:130.

27. Hoebel BG, Avena NM, Rada P. Accumbens dopamine-acetylcholine balance in approach and avoidance. Curr Opin Pharmacol. 2007;7:617-27. 
28. Lester DB, Rogers TD, Blaha CD. Acetylcholine-Dopamine Interactions in the Pathophysiology and Treatment of CNS Disorders. CNS Neurosci Ther. 2010;16:137-62.

29. Raczka KA, Mechias ML, Gartmann N, Reif A, Deckert J, Pessiglione M, Kalisch R. Empirical support for an involvement of the mesostriatal dopamine system in human fear extinction. Translational Psychiatry. 2011;1:e12-2.

30. Huang D, Xu J, Wang J, Tong J, Bai X, Li H, Wang Z, Huang Y, Wu Y, Yu M, Huang F. Dynamic Changes in the Nigrostriatal Pathway in the MPTP Mouse Model of Parkinson's Disease. Parkinson's Disease. 2017; 2017:1-7.

31. Kordower JH, Olanow CW, Dodiya HB, Chu Y, Beach TG, Adler CH, Halliday GM, Bartus RT. Disease duration and the integrity of the nigrostriatal system in Parkinson's disease. Brain. 2013;136:241931.

32. Furukawa $\mathrm{H}$, Gouaux E. Mechanisms of activation, inhibition and specificity: crystal structures of the NMDA receptor NR1 ligand-binding core. EMBO J. 2003;22(12):2873-85.

33. Hansen KB, Yi F, Perszyk RE, Menniti FS, Traynelis SF. NMDA Receptors in the Central Nervous System. 2017;1677:1-80.

34. Hansen KB, Yi F, Perszyk RE, Furukawa H, Wollmuth LP, Gibb AJ, Traynelis SF. Structure, function, and allosteric modulation of NMDA receptors. J Gen Physiol. 2018;150:1081-105.

35. Kim N, Chen D, Zhou XZ, Lee TH. Death-Associated Protein Kinase 1 Phosphorylation in Neuronal Cell Death and Neurodegenerative Disease. Int J Mol Sci. 2019;20:3131.

36. Wang S, Shi X, Li H, Pang P, Pei L, Shen H, Lu Y. DAPK1 Signaling Pathways in Stroke: from Mechanisms to Therapies. Mol Neurobiol. 2017;54:4716-22.

37. Wilbek TS, Skovgaard T, Sorrell FJ, Knapp S, Berthelsen J, Stromgaard K. Identification and characterization of a small-molecule inhibitor of death-associated protein kinase 1. Chembiochem. 2015;16:59-63.

38. Chen D, Zhou XZ, Lee TH. Death-Associated Protein Kinase 1 as a Promising Drug Target in Cancer and Alzheimer's Disease. Recent Pat Anticancer Drug Discov. 2019;14:144-57.

\section{Figures}



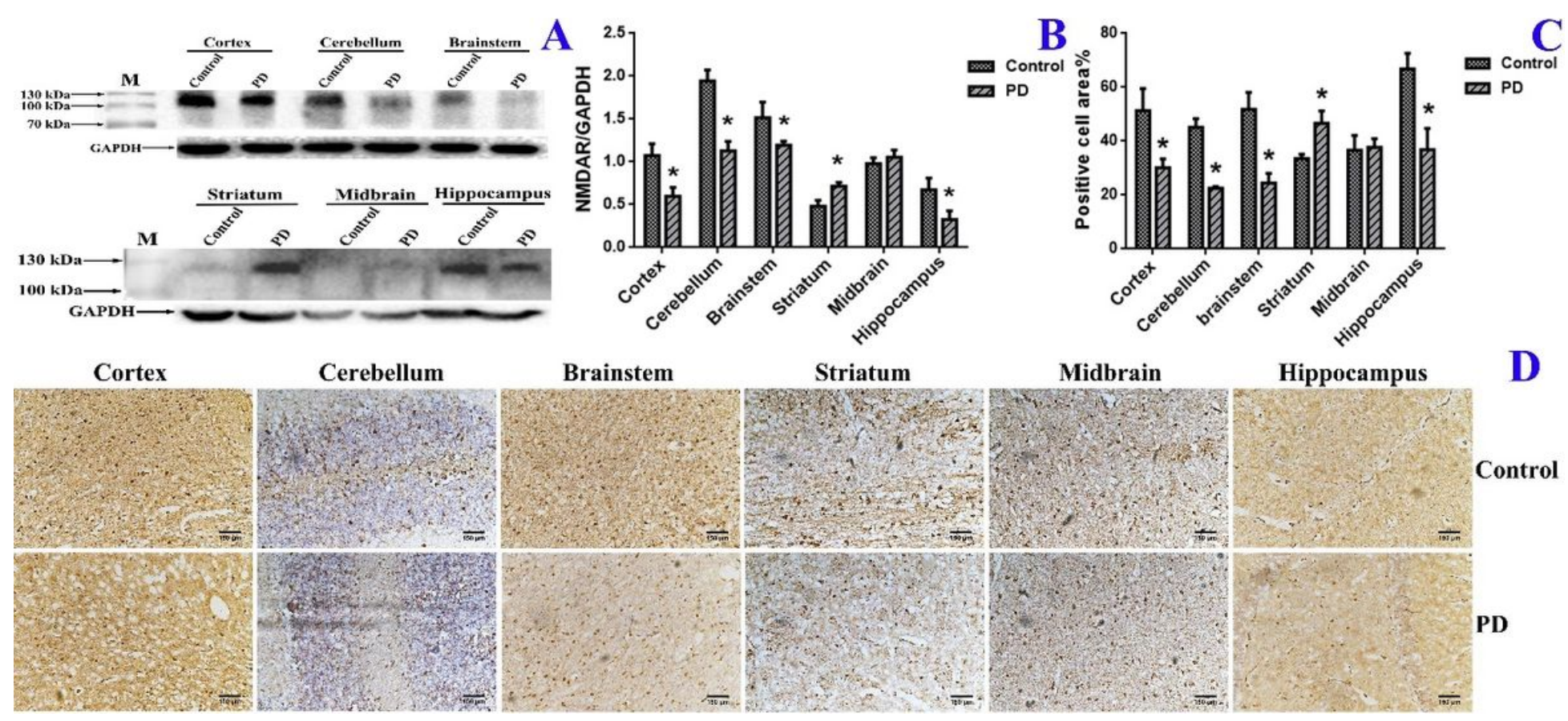

\section{Figure 1}

A tissue-specific analysis of NMDAR1 abundance in a various specialized structures of mouse brains. a Western blot analysis on NMDAR1. One hundred and twenty microgram of total protein was subjected to Western blot analysis coupled with $10 \%$ SDS-PAGE separation. The visualization of protein bands was conducted using ECL. M, PageRuler prestained protein ladder (Fermentas). b Densitometric analysis of NMDAR1 normalized to the level of GAPDH. The statistical analyses were conducted by a two-tailed equal variance Student's t-test. Bars represented the mean $\pm S E M ; *, P<0.05$. c Statistical evaluation of NMDAR1 analyzed by IHC. The analyses were performed by a two-tailed equal variance Student's t-test; Bars represented the mean $\pm \mathrm{SEM}$; ${ }^{*}, \mathrm{P}<0.05$. $\mathrm{d}$ Immunohistochemical staining of NMDAR1 (at magnification of 400). The sections were immunostained with monoclonal anti-NMDAR1 antibody, counterstained with hematoxylin, and visualized by DAB. 


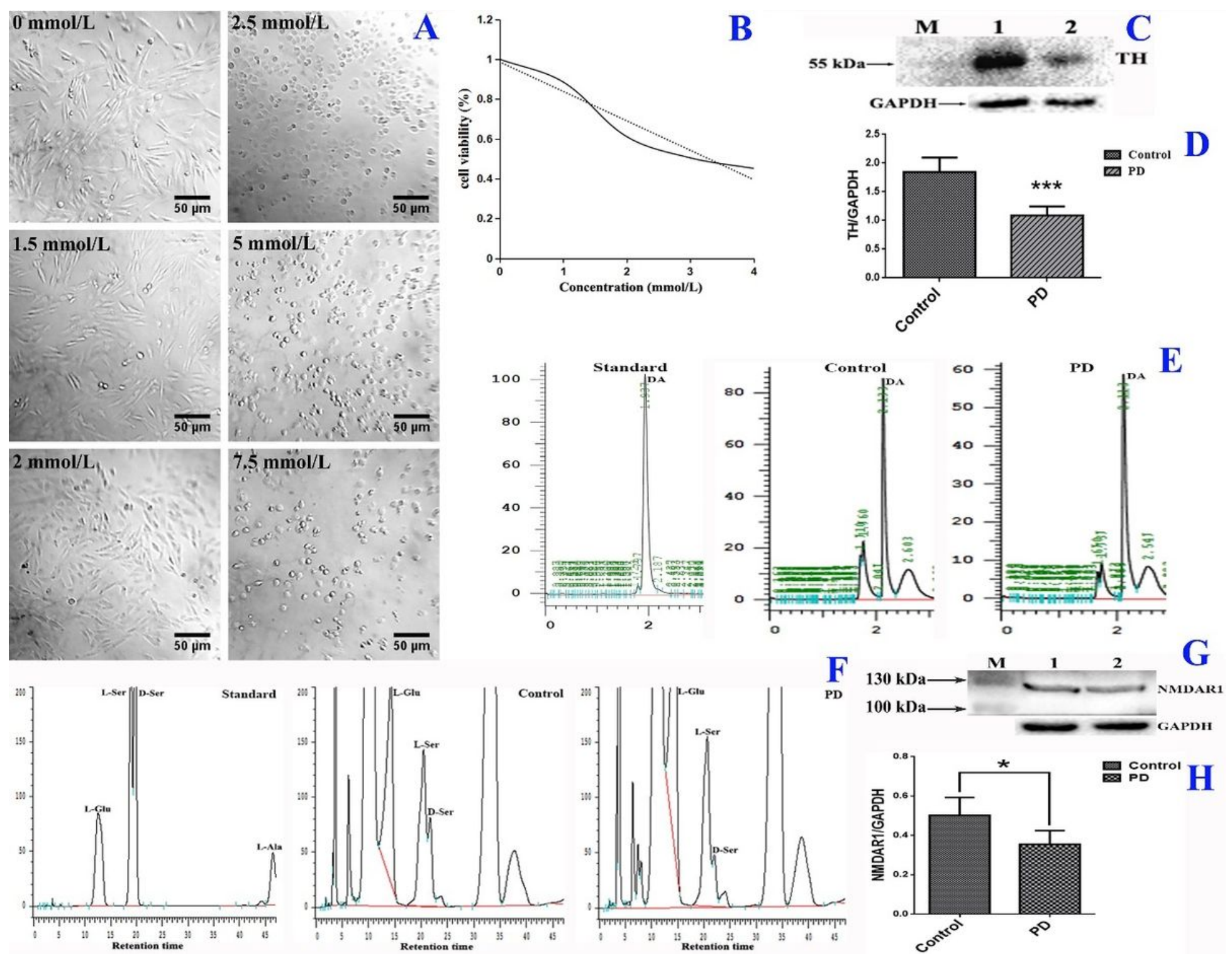

Figure 2

Characterization of the rat C6 glioma cells with a PD-like variation. M, PageRuler prestained protein ladder (Fermentas); 1, untreated cells; 2, MPP+-treated cells. a, Morphological observation of the rat C6 glioma cells. The images were photographed at magnification of 200 under light microscopy. Scale bar represented $50 \mu \mathrm{m}$. b, Effect of MPP+ on cell viability of the rat C6 glioma cells using MTT assay. c, Representative image of TH expression analyzed by Western blot. The samples were blotted with monoclonal anti-TH antibody, and protein bands were visualized using ECL kit. d, Densitometric analysis of TH normalized to the level of GAPDH. The statistical analyses were conducted by a two-tailed equal variance Student's t-test. Values were expressed as the mean $\pm S E M ; *, P<0.05$. e, Representative HPLC analysis on cellular DA and its standard. $f$, Representative HPLC analysis on cellular D-serine and standards. g, Representative image of cellular NMDAR1 abundance analyzed by Western blot. The samples were blotted with monoclonal anti-NMDAR1 antibody, and protein bands were visualized using ECL kit. h, Densitometric analysis of NMDAR1 normalized to the level of GAPDH. The statistical analyses 
were conducted by a two-tailed equal variance Student's t-test. Values were expressed as the mean $\pm S E M$; *, $\mathrm{P}<0.05$.
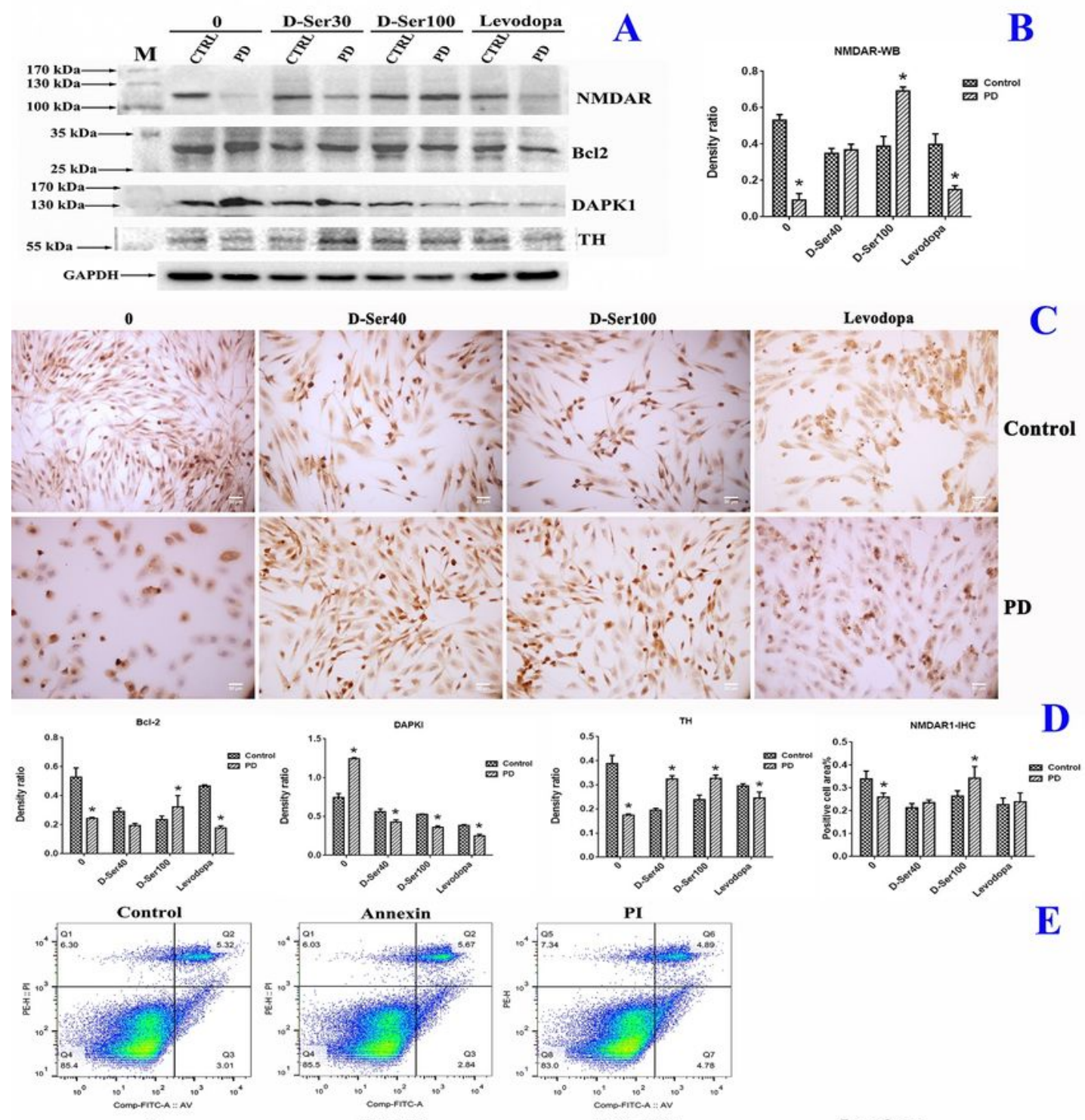

$\mathbf{E}$
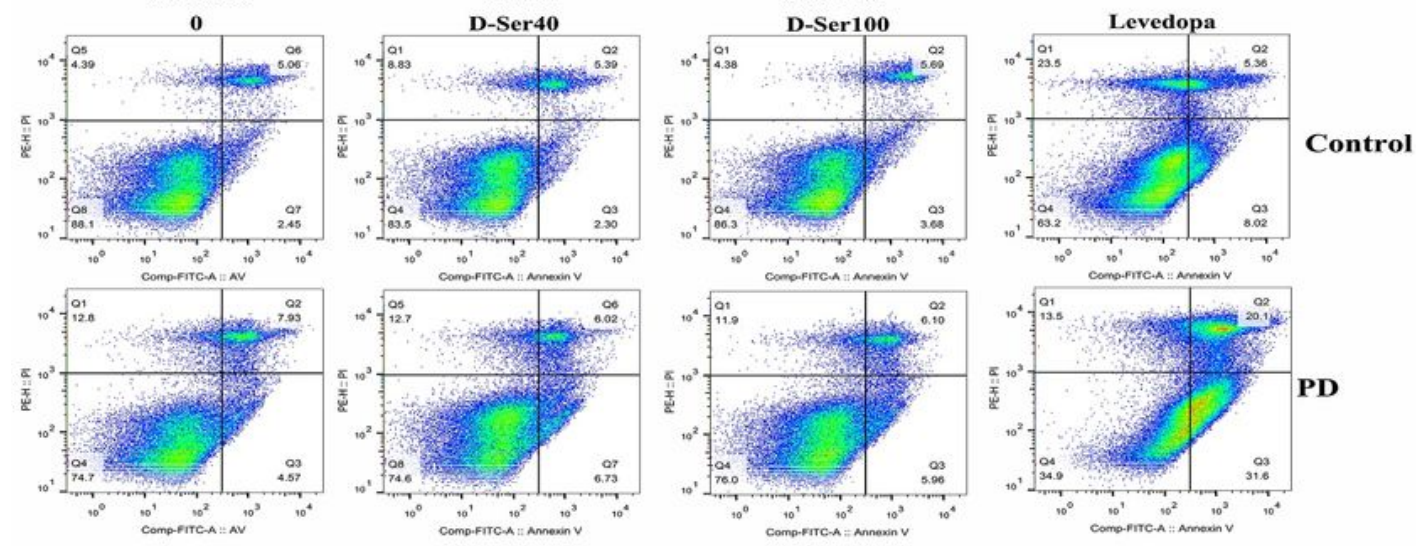

Figure 3

Effect of D-Serine on molecules associated with representative parkinsonian parameters and DAPKIassociated signal transduction pathway. a Representative photographs of Western blot analysis on NMDAR1, Bcl2, DAPK1 and TH in the rat C6 glioma cell. The membranes were blotted with monoclonal 
anti-NMDAR1 antibody, anti-Bcl2 antibody, anti-DAPK1 antibody and anti-TH antibody, respectively. Visualization of protein bands was performed using ECL kit. M, PageRuler prestained protein ladder (Fermentas). b Quantification of NMDAR1 bands analyzed by Western blot. Protein bands were statistically analyzed by Image $\mathrm{J}$ with background subtraction. c Representative micrographs of ICC taken at the magnification of 400 . The cells were blotted with monoclonal anti-NMDAR1 antibody, stained with $D A B$, and counterstained with hematoxylin. d Quantification of protein bands analyzed by Western blot (including Bcl2, DAPK1, TH) and statistic analysis of NMDAR1 expression detected by ICC. Protein bands were statistically analyzed by Image $\mathrm{J}$ with background subtraction. ICC images was statistically evaluated using Image Pro-plus. The statistic figures were plotted using Graphpad Prism (version 6.0). The data were expressed as the mean $\pm S E M ; *, P<0.05$. e Evaluation of cell death and apoptosis in the rat C6 glioma cells using AnnexinV-FITC/PI staining and flow cytometry.
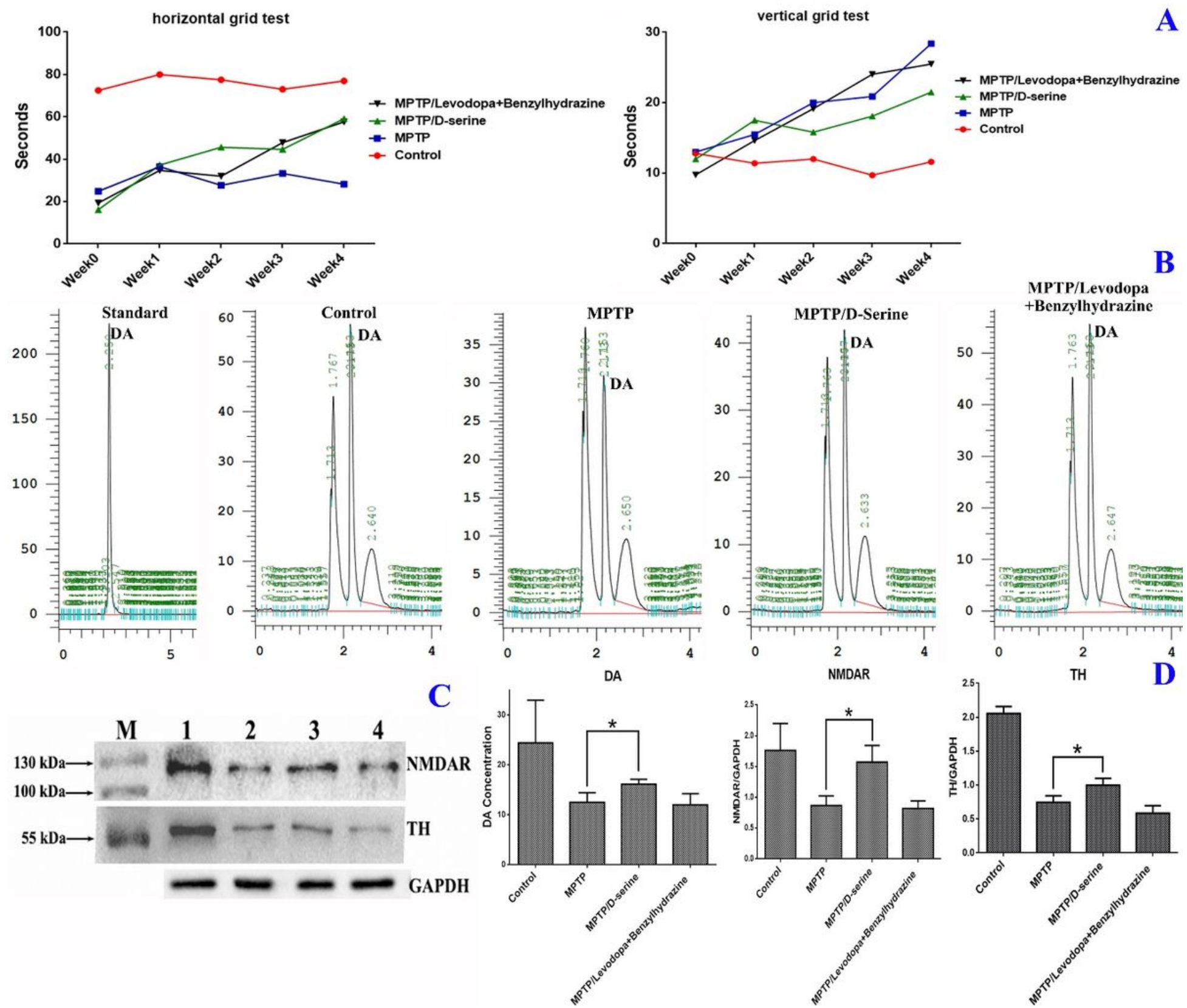

Figure 4 
Verification of the essential parkinsonian symptoms in mice. a) Verification of behavioral activity in the mice with PD-like symptoms. b) Representative HPLC analysis on striatal DA and its standard. (c) Representative photographs of Western blot analysis on NMDAR1 and TH in the striatum of the D-serinetreated mice. The membranes were blotted with monoclonal anti-NMDAR1 antibody and anti-TH antibody, respectively. d) Statistical analysis of Western blot staining for NMDAR1 and TH between the Dserine-treated mice and their controls. The statistic analyses were conducted by a two-tailed equal variance Student's t-test; bars represented the mean $\pm \mathrm{SEM}$; ${ }^{*}, \mathrm{P}<0.05$.
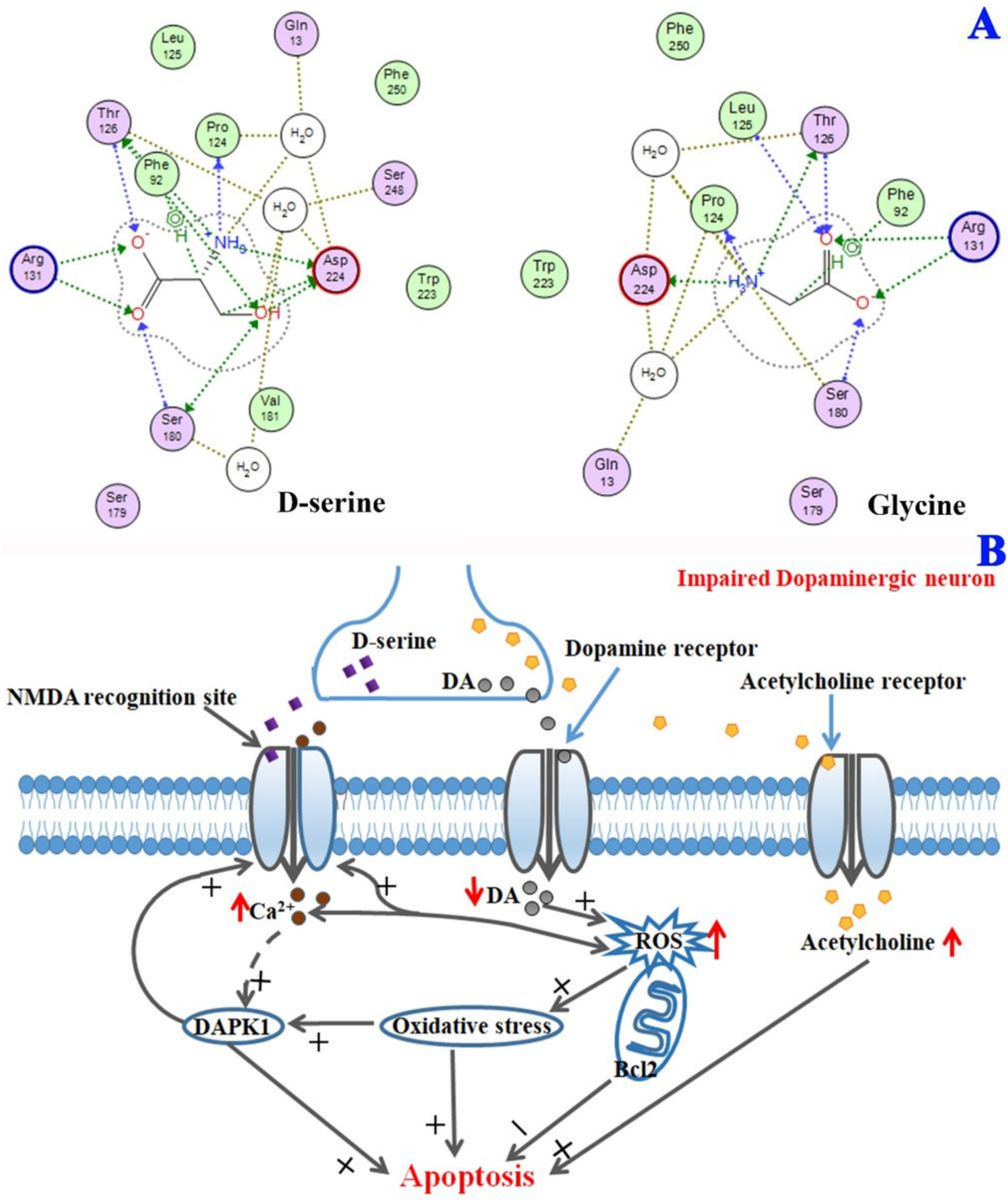


\section{Figure 5}

Evaluation of ligand interactions of NMDAR1 with D-serine/glycine, and possible signal transduction pathway involved in D-serine/NMDAR1. (a) Schematic 2D views of the interactions between Dserine/glycine and binding pocket residues created by the Ligand Interactions module of the MOE. (b) Schematic signal transduction pathway involved in NMDAR1. Red, in impaired neuronal cells. +, promote; -, inhibit; , down-regulated; , up-regulated.

\section{Supplementary Files}

This is a list of supplementary files associated with this preprint. Click to download.

- FigureS1cellularmorphology2.tif

- FigureS2ApoptosisHoechast1.tif 